\title{
Impact of the LMDZ atmospheric grid configuration on the climate and sensitivity of the IPSL-CM5A coupled model
}

\author{
Frédéric Hourdin • Marie-Alice Foujols • Francis Codron • Virginie Guemas • \\ Jean-Louis Dufresne - Sandrine Bony • Sébastien Denvil • Lionel Guez • \\ François Lott • Josefine Ghattas • Pascale Braconnot • Olivier Marti • \\ Yann Meurdesoif • Laurent Bopp
}

Received: 24 October 2011/ Accepted: 28 May 2012/Published online: 26 July 2012

(C) The Author(s) 2012. This article is published with open access at Springerlink.com

\begin{abstract}
The IPSL-CM5A climate model was used to perform a large number of control, historical and climate change simulations in the frame of CMIP5. The refined horizontal and vertical grid of the atmospheric component, LMDZ, constitutes a major difference compared to the previous IPSL-CM4 version used for CMIP3. From imposedSST (Sea Surface Temperature) and coupled numerical experiments, we systematically analyze the impact of the horizontal and vertical grid resolution on the simulated climate. The refinement of the horizontal grid results in a systematic reduction of major biases in the mean tropospheric structures and SST. The mid-latitude jets, located too close to the equator with the coarsest grids, move poleward. This robust feature, is accompanied by a drying at mid-latitudes and a reduction of cold biases in mid-latitudes relative to the equator. The model was also extended to the stratosphere by
\end{abstract}

This paper is a contribution to the special issue on the IPSL and CNRM global climate and Earth System Models, bothdeveloped in France and contributing to the 5th coupled model intercomparison project.

F. Hourdin ( $($ )

Laboratoire de Météorologie Dynamique, (LMD/IPSL),

CNRS-UPMC-ENS-EP, Tr 45-55, 3e et, B99 Jussieu,

Paris 75005, France

e-mail: hourdin@1md.jussieu.fr

M.-A. Foujols $\cdot$ S. Denvil · J. Ghattas

Institut Pierre-Simon Laplace (IPSL), CNRS-UPMC,

UVSQ, CEA, Paris, France

F. Codron $\cdot$ V. Guemas $\cdot$ J.-L. Dufresne $\cdot$ S. Bony

L. Guez · F. Lott

LMD, Paris, France

P. Braconnot - O. Marti · Y. Meurdesoif · L. Bopp

Laboratoire des Sciences du Climate et de l'Environnement

(LSCE/IPSL), CNRS-CEA-UVSQ, Saclay, France increasing the number of layers on the vertical from 19 to 39 (15 in the stratosphere) and adding relevant parameterizations. The 39-layer version captures the dominant modes of the stratospheric variability and exhibits stratospheric sudden warmings. Changing either the vertical or horizontal resolution modifies the global energy balance in imposedSST simulations by typically several $\mathrm{W} / \mathrm{m}^{2}$ which translates in the coupled atmosphere-ocean simulations into a different global-mean SST. The sensitivity is of about $1.2 \mathrm{~K}$ per $1 \mathrm{~W} / \mathrm{m}^{2}$ when varying the horizontal grid. A re-tuning of model parameters was thus required to restore this energy balance in the imposed-SST simulations and reduce the biases in the simulated mean surface temperature and, to some extent, latitudinal SST variations in the coupled experiments for the modern climate. The tuning hardly compensates, however, for robust biases of the coupled model. Despite the wide range of grid configurations explored and their significant impact on the present-day climate, the climate sensitivity remains essentially unchanged.

Keywords Climate modeling - Grid resolution . Climate change projections

\section{Introduction}

Numerical simulations with general circulation models are at the heart of climate change studies. They are used to quantify the impact of greenhouse gas increase on the evolution of the global climate, to unravel the physical mechanisms that control climate sensitivity, and to verify theoretical hypotheses or mechanisms while taking into account the complexity of the climate system. Those numerical models however still provide only an approximate representation of the real climate system, which 
constitutes a major source of uncertainty for assessing future climate changes. Improving the models should therefore be one of the main drivers of climate research.

Among the limitations often emphasized is the rather coarse spatial resolution of the models used for long-term climate change simulations, such as those coordinated by the Coupled Model Intercomparison Project (CMIP, Meehl et al. 2007; Taylor et al. 2012). It is partly because of this coarse resolution that key processes such as convection or clouds have to be parameterized. Systematic centennial global simulations with meshes of the order of $50 \mathrm{~m}$, which would be required to explicitly represent boundary layer clouds, will not be reachable before at least a couple of decades. It is however expected that significant improvements can already be achieved by increasing the spatial resolution of current climate models from a few hundreds to a few tens of kilometers, both because it allows a better resolution of the dominant atmospheric large scale dynamics and because it offers a finer description of surface conditions (orography, land/sea distribution). Among the expected improvements are a reduction of systematic biases in temperature, precipitation and winds (Pope and Stratton 2002; Roeckner et al. 2006; Hack et al. 2006), a better representation of the regional-scale climate (Williamson et al. 1995; Kobayashi and Sugi 2004; Navarra 2008; Byrkjedal et al. 2008), and a better representation of rainfall distributions (Kiehl and Williamson 1991; Déqué et al. 1994). An important question in the frame of climate change simulations is to know whether the model limitations, and in particular the biases which come from the use of coarse grids, impact the climate sensitivity, both in a global sense and in modifications of the climate regimes.

Within the framework of the preparation of the 5th phase of CMIP (CMIP5, Taylor et al. 2012) at the Institut Pierre-Simon Laplace (IPSL), a systematic exploration of the impacts of changes in the atmospheric grid configuration of the LMDZ atmospheric general circulation model was conducted. The simulations were performed with the LMDZ4 version (Hourdin et al. 2006), the atmospheric component of the IPSL Coupled Model IPSL-CM4 (Braconnot et al. 2007; Marti et al. 2010) that took part in CMIP3 (Meehl et al. 2007). The results of this systematic exploration were used to choose the final configuration LMDZ5A, the atmospheric component of the IPSL-CM5A model used for CMIP5. Since we intended to contribute to CMIP5 with a wide variety of configurations and ensembles of simulations (Dufresne et al., this issue), rather coarse resolutions were explored.

One major goal of this comparison of different grids was to understand how model biases evolve with increasing resolution. It appears that grid refinement affects the position of the jets, and in turn the mid-latitude cold bias which was one of the major deficiencies of IPSL-CM4. The cause of the impact of grid refinement on the jet latitude is found in large-scale atmospheric dynamics, and was studied by Guemas and Codron (2011). Here we show that these changes also affect significantly the biases of the coupled model, as well as the mean climate equilibrium temperature.

Research over the last decades have led to an increasing recognition of the role of the stratosphere in controlling some aspects of the tropospheric climate. This influence is related to radiative and chemical effects, but also to dynamical effects: some modes of stratospheric variability propagate downward, like the Quasi-Biennial Oscillation (QBO, Baldwin et al. 2001) in the tropics, and the Arctic Oscillation (AO, Baldwin and Dunkerton 1999) in the mid latitudes. When the stratospheric anomalies reach the tropopause, they can potentially influence the surface climate, at least in the mid-latitudes (for the AO effect in the LMDZ mid-latitudes see for instance Lott et al. 2005; Nikulin and Lott 2010). In order to take into account the impact of the stratospheric dynamics and chemistry in the coupled climate simulations, the LMDZ vertical grid was extended in the stratosphere, with a resolution close to a previous stratospheric version of LMDZ4 described by Lott et al. (2005). After these changes the model can be considered as a high-top climate model.

The results and discussions of the present paper are mainly focused on the impact of the configuration changes on the model biases and climate sensitivity. It is shown in particular that despite a significant impact on some biases in the present-day climate, the climate sensitivity is weakly affected by the changes in grid configuration. Additional results concerning the impact of changes in grid configuration are discussed in companion papers in the same issue: the impact of the refinement of the horizontal grid on the atmospheric variability in the north-Atlantic region is discussed by Cattiaux et al. and results on the ENSO variability are shown by Dufresne et al. in an overview paper of the IPSL-CM5 model. ${ }^{1}$

The paper is organized as follows. In Sect. 2, the consequences of the model horizontal grid refinement on the mean climatology and on the latitudinal structure in the LMDZ4 simulations with imposed SSTs, and in the coupled atmosphere-ocean simulations with IPSL-CM4, are documented and analyzed. Section 3 is dedicated to the impact of the vertical extension of the model to the stratosphere. Finally, we compare in Sect. 4 the mean climate and the climate sensitivity to an increase in greenhouse gases of the configurations of the IPSL coupled model involved in the CMIP3 and CMIP5 exercises.

\footnotetext{
1 The drafts of the special issue papers can be found at http://icmc.ipsl.fr/research/international-projects/cmip5/special-issuecmip5.
} 


\section{Refining the horizontal grid in LMDZ4 and IPSL-CM4 simulations}

We analyze in this section a series of imposed-SST and coupled atmosphere-ocean simulations, all done with the same LMDZ4 atmospheric model, but with varying horizontal grids.

\subsection{The LMDZ4 general circulation model}

LMDZ is an atmospheric general circulation model developed at Laboratoire de Météorologie Dynamique. The dynamical part of the code is based on a finite-difference formulation of the primitive equations of meteorology (see e.g. Sadourny and Laval 1984), discretized on a stretchable ( $\mathrm{Z}$ of LMDZ standing for Zoom capability) longitude-latitude Arakawa C-grid.

The physical parameterizations of the LMDZ4 versions are described by Hourdin et al. (2006). The Morcrette (1991) scheme is used for radiative transfer. Drag and lifting effects associated with the subgrid-scale orography are accounted for according to Lott (1999). Turbulent transport in the planetary boundary layer is treated as a vertical diffusion with an eddy diffusivity $K_{z}$ depending on the local Richardson number according to Laval et al. (1981). Up-gradient transport of heat in the convective boundary layer is ensured by adding a prescribed countergradient term of $1 \mathrm{~K} / \mathrm{km}$ to the vertical derivative of potential temperature (Deardorff 1966). In the case of unstable profiles, a dry convective adjustment is applied. The surface boundary layer is treated according to Louis (1979). Deep convection is parameterized using the "episodic mixing and buoyancy sorting" Emanuel scheme (Emanuel 1991) which assumes quasi-equilibrium between the opposite influences of the large-scale forcing of convection and of convective instability. A statistical cloud scheme is used to predict the clouds properties with a different treatment for convective clouds (Bony and Emanuel 2001) and large-scale condensation as explained by Hourdin et al. (2006).

The IPSL-CM4 simulations made for CMIP3 were performed with a configuration of LMDZ4 made of 96 points in longitude by 72 in latitude (about $3.75^{\circ} \times 2.5^{\circ}$ ) and 19 layers on the vertical (Marti et al. 2010).

\subsection{Sensitivity experiments}

Identical changes in horizontal resolution are explored here in both imposed-SST and coupled atmosphere-ocean simulations with exactly the same source code for the atmospheric component LMDZ4, using a 19-layer vertical grid (L19). The dynamical time-step and the time constants for the horizontal diffusion are the only-necessary- parameter changes between the different simulations, as described below. The other components of the system, i.e. the land surface scheme Orchidee and the oceanic circulation model Nemo, are also strictly identical (those versions are described by Marti et al. 2010).

In the imposed-SST simulations, seasonally varying SSTs are imposed as a boundary condition. In practice, a climatological average of the AMIP SSTs (Hurrell et al. 2008) over the period 1970-2000 is used in order to minimize the number of years of simulation required to smooth out the inter-annual variability. The forced simulations are run for 10 years.

For the coupled atmosphere-ocean simulations, we show results of control simulations in which the concentration of greenhouse gases, the Earth's orbital parameters and solar irradiance, and aerosols are kept constant, with same values as in the imposed-SST experiments. The model is run for 100 years. The control simulations are analyzed after a spin-up phase so that the global radiative balance is within $1 \mathrm{~W} / \mathrm{m}^{2}$ from zero in all the simulations. For the illustrations below, the climatological mean seasonal cycle is computed from the last 10 years of the simulations.

LMDZ uses for the time integration a leapfrog scheme with a Matsuno (or forward/backward) step every five leapfrog time-steps. The time step $\delta t$ is limited by a CFL criteria, which varies linearly with the size $\delta x_{\min }$ of the smallest grid cell: $\delta t<\delta x_{\min } / C$, where the $C$ constant is the external gravity waves phase speed in the model. In longitude-latitude grids, the longitudinal grid size goes to zero at the pole. In order to avoid the use of too small time-steps, a longitudinal filter is applied to the dynamical equations after latitude $\phi_{0}=60^{\circ}$ in both hemispheres. For a regular longitude-latitude grid as used here, the minimum scale explicitly accounted for in $x$ is $\delta x_{\min }=\delta x_{\max } * \cos \left(\phi_{0}\right)=\delta x_{\max } / 2$, where $\delta x_{\max }=2 \pi a / I M$ is the mesh size in $x$ at the equator, $a=6,400 \mathrm{~km}$ being the Earth radius and $I M$ the number of grid cells in the longitudinal direction. Poleward of the latitude $\phi_{0}$, meteorological fields are filtered so as to retain only wave lengths longer than $\delta x_{\min }$. The grid mesh size in latitude $\delta y=\pi a / J M$ - where $J M$ is the number of points in latitude is a constant for a given grid. Finally, the time step is limited by $\delta t<(\pi a / C) \min (1 / I M, 1 / J M)$.

In a longitude-latitude grid, the isotropy of the horizontal grid $(\delta y=\delta x)$ cannot be insured everywhere. The original grid, $(I M, J M)=(96,72)$, or $(d \lambda, d \phi)=$ $\left(3.75^{\circ}, 2.5^{\circ}\right)$, has a ratio $I M / J M=4 / 3$ chosen so that the grid is isotropic at close to $45^{\circ}$ latitude. This choice yields $\delta x=3 \delta y / 2$ at the equator; and the time step is limited by $\delta x$ at $\phi_{0}$. Keeping both the same resolution in longitude and the same value of $\phi_{0}=60^{\circ}$, it is possible to refine further the resolution in latitude up to $J M=I M$ without reducing the time step. The grid is then isotropic at $60^{\circ}$ latitude, and $\delta x / \delta y=2$ at the equator. 
The simulations presented here were performed with either $J M=3 / 4 \times I M$ or $J M=I M$. Resolutions from $(d \lambda, d \phi)=\left(3.75^{\circ}, 2.5^{\circ}\right)$ to $\left(1.875^{\circ}, 1.26^{\circ}\right)$ or $(I M, J M)=$ $(96,71)$ to $(192,142)$ were explored with the coupled atmosphere-ocean model, by increasing successively either the latitudinal or longitudinal resolution. The same resolutions as well as finer grids were explored with the imposed-SST model. Characteristics of the simulations are given in Table 1.

In addition to the choice of a small enough time step, the numerical stability of the model is ensured by the "horizontal dissipation" operators. Those operators account for the interaction between the explicit and sub-grid scales. They are also crucial for numerical stability. Without dissipation, the enstrophy cascade-well represented in LMDZ which favors numerical conservation of enstrophy (Sadourny 1975)_would accumulate at the cut-off scale. The efficiency of those operators is controlled by two constants: the number of iterations $\mathcal{N}$ and a time constant $\tau$. The larger the value of $\mathcal{N}$, the more scale-selective the operator is, the e-folding time of an oscillation of wavenumber $k$ scaling with $k^{2 \mathcal{N}}$. The time constant $\tau$ is the e-folding time of the largest value of $k$ encountered in the $\operatorname{mesh}\left(k_{\max } \sim 1 / \delta x_{\min }\right)$. In practice, a Laplacian operator is used for the lateral diffusion of potential temperature, while the vector Laplacian used for wind dissipation is divided into rotational and divergent components. $\mathcal{N}=2$ is used for the temperature and the wind rotational, with the same time constant $\tau_{1}$. For the wind divergence, a stronger dissipation is applied by using both $\mathcal{N}=1$ and a shorter time constant $\tau_{2}<\tau_{1}$. In practice, the time constants are slightly adjusted (reduced) empirically to insure numerical stability when refining the grid. Note however that the effective diffusivity at a given scale decreases drastically when refining the grid. The retained values are given in Table 1.

\subsection{SST cold biases and dynamical structure}

One of the major deficiencies of the IPSL-CM4 CMIP3 simulations was a strong cold bias in the mid-latitude SSTs, in both the Northern and Southern hemispheres (Swingedouw et al. 2007; Marti et al. 2010). The zonal-mean bias reaches $4 \mathrm{~K}$ around $40^{\circ}$ of latitude for the IPSL-CM4 $96 \times 71$ simulation (Fig. 1). Refining the resolution in latitude significantly reduces this bias. With a refinement in longitude, the warming of the model is essentially located in the tropics, as illustrated further in Fig. 2. The equator-tomid-latitude surface temperature contrast is generally 0.5-1 K smaller (and thus closer to observations) when the same number of points is used in longitude and latitude. The bias in the equator-to-mid-latitude contrast is of $4 \mathrm{~K}$ for the $96 \times 71$ simulation, which has nearly zero SST-bias at the equator. It reduces down to $2 \mathrm{~K}$ in the $144 \times 142$ case, but increases back to $3 \mathrm{~K}$ for the $192 \times 142$ grid.

The reduction of the cold bias of the mid-latitudes when refining the grid is accompanied by a poleward shift of the mid-latitude jets (Fig. 3). This shift is present both in the IPSL-CM4 coupled and LMDZ4 imposed-SST simulations. It corresponds to a strong reduction of the biases in

Table 1 Characteristics of the model configurations used for this study

\begin{tabular}{|c|c|c|c|c|c|c|c|c|c|c|}
\hline $\begin{array}{l}\text { Horizontal grid } \\
(I M \times J M)\end{array}$ & $\begin{array}{l}\text { CMIP config. } \\
\text { IPSL- }\end{array}$ & $\begin{array}{l}d \lambda \times d \phi \\
\left({ }^{\circ} \times{ }^{\circ}\right)\end{array}$ & $\begin{array}{l}\delta x \times \delta y(\mathrm{~km} \times \mathrm{km}) \\
\text { at } 45^{\circ}\end{array}$ & $\begin{array}{l}\delta t \\
(\mathrm{~s})\end{array}$ & $\begin{array}{l}\tau_{1} \\
\text { (hrs) }\end{array}$ & $\begin{array}{l}\tau_{2} \\
\text { (hrs) }\end{array}$ & $\begin{array}{l}\text { Imposed } \\
\text { SST }\end{array}$ & Control & Historical & $\begin{array}{l}1 \% \\
\mathrm{CO}_{2}\end{array}$ \\
\hline \multicolumn{11}{|c|}{ LMDZ4, IPSL-CM4, L19, CMIP3 } \\
\hline $96 \times 71$ & CM4 & $3.75 \times 2.5$ & $296 \times 280$ & 180 & 2.0 & 1.5 & $\mathrm{X}$ & $\mathrm{X}$ & & $\mathrm{X}$ \\
\hline $96 \times 95$ & & $3.75 \times 1.9$ & $296 \times 209$ & 180 & 2.0 & 1.5 & $\mathrm{X}$ & $\mathrm{X}$ & & $\mathrm{X}$ \\
\hline $144 \times 95$ & & $2.5 \times 1.9$ & $197 \times 209$ & 120 & 2.0 & 1.5 & $\mathrm{X}$ & $\mathrm{X}$ & & \\
\hline $144 \times 142$ & & $2.5 \times 1.25$ & $197 \times 140$ & 120 & 1.5 & 1.0 & $\mathrm{X}$ & $\mathrm{X}$ & & $\mathrm{X}$ \\
\hline $192 \times 142$ & & $1.88 \times 1.25$ & $148 \times 140$ & 90 & 1.5 & 1.0 & $\mathrm{X}$ & $\mathrm{X}$ & & \\
\hline $192 \times 192$ & & $1.88 \times 0.93$ & $148 \times 104$ & 90 & 1.5 & 1.0 & $\mathrm{X}$ & & & \\
\hline $280 \times 192$ & & $1.29 \times 0.93$ & $101 \times 104$ & 60 & 1.5 & 1.0 & $\mathrm{X}$ & & & \\
\hline \multicolumn{11}{|c|}{ LMDZ5A, IPSL-CM5A, L39, CMIP5 } \\
\hline $96 \times 95$ & CM5A-LR & $3.75 \times 1.9$ & $296 \times 209$ & 180 & 1.5 & 1.5 & $\mathrm{X}$ & $\mathrm{X}$ & $\mathrm{X}$ & $\mathrm{X}$ \\
\hline $144 \times 142$ & CM5A-MR & $2.5 \times 1.25$ & $197 \times 140$ & 120 & 1.5 & 1.0 & $\mathrm{X}$ & $\mathrm{X}$ & $\mathrm{X}$ & $\mathrm{X}$ \\
\hline
\end{tabular}

The IPSL-CM4 model used for CMIP3 was based on the $96 \times 71$ horizontal grid configuration of the LMDZ4 atmospheric general circulation model with 19 layers on the vertical (L19). A series of sensitivity experiments to the horizontal grid was performed with the same model version. For CMIP5, the IPSL-CM5A model (LMDZ5A atmospheric component with 39 layers) was run both with a low resolution (LR, 96 $\times 95)$ and mid resolution $(\mathrm{MR}, 144 \times 142)$ grid. $\delta t$ is the time-step used for primitive equations integration. The physical package is called with a time-step of $30 \mathrm{~min}$ for all the model configurations. The radiative transfer is computed each two hour for the IPSL-CM4 simulations and every hour for IPSL-CM5A-LR and -MR. $\tau_{1}$ and $\tau_{2}$ are the time constants for horizontal dissipation. The last four columns indicate the simulations used in the present study. See text for further explanations 


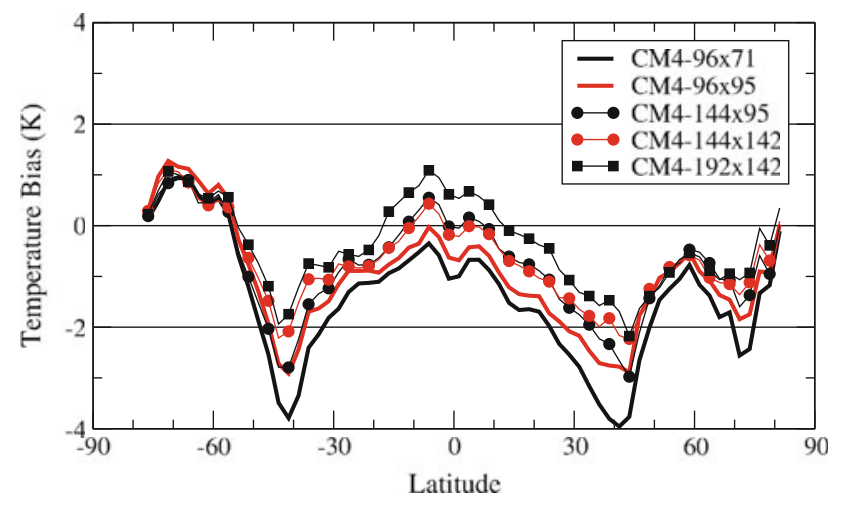

Fig. 1 Biases in SST (K) for the various configurations of the IPSLCM4 model. The biases are computed with respect to the Levitus climatology, and zonally averaged. We analyze the last 10 years of 100 -year simulations starting from the same oceanic state. The red curves correspond to cases where $I M=J M$

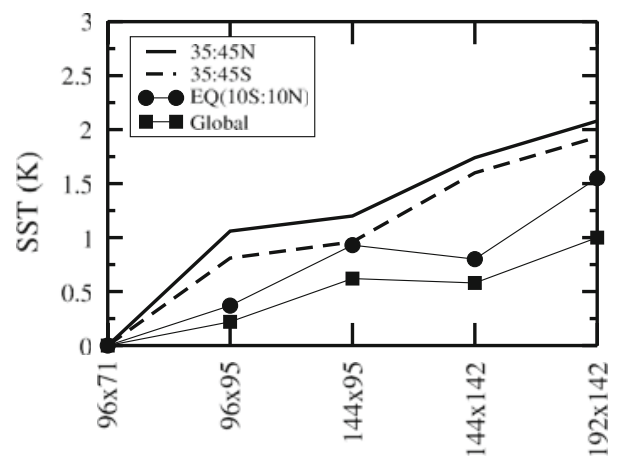

Fig. 2 Evolution with the model horizontal resolution of the SST (K) for the global average (squares), for the southern (45-35S, dashed line) and northern (35-45N, full line) mid-latitudes and for the Equator $(5 \mathrm{~S}-5 \mathrm{~N}$, circles). The values from the coarsest grid $(96 \times 71)$ are subtracted

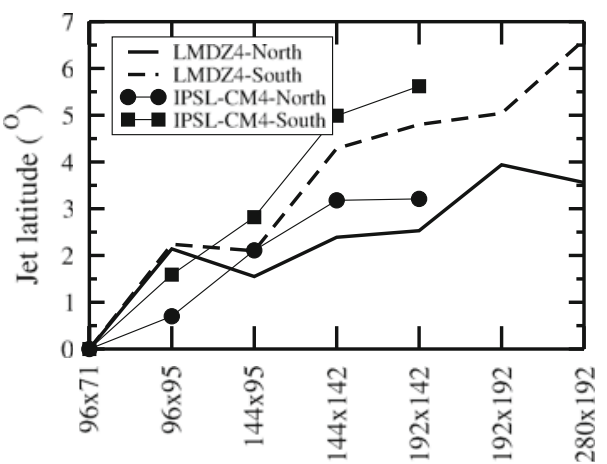

Fig. 3 Latitude of the mid-latitude jets, computed at the $850 \mathrm{hPa}$ level, for the two hemispheres and for the imposed-SST (LMDZ4) and coupled atmosphere-ocean (IPSL-CM4) simulations. The latitude is counted positive from equator to pole in both hemispheres and the values from the coarsest $(96 \times 71)$ grid are subtracted

the representation of the mean zonal wind with grid refinement, as illustrated in the left column of Fig. 4 for the imposed-SST simulations. For the coarsest grids, the jets are shifted toward the equator compared to ERA interim reanalyzes (as seen from the strong dipole in the zonal wind bias, centered at the latitude of the jet maximum intensity).

This jet displacement was studied by Guemas and Codron (2011) in a set of dynamical core experiments produced with the LMDZ atmospheric model using the Held and Suarez (1994) setup. This setup consists in replacing all the detailed physical parameterizations by a Newtonian relaxation of the temperature field toward a zonally-symmetric state, and a Rayleigh (linear) damping of the low-level wind with an e-folding timescale of 1 day at the surface. In this configuration, it was shown that the jet latitude moves poleward when refining the grid in latitude, and is less affected when increasing the number of grid points in longitude. It was checked also in this idealized framework that the changes in jet location when refining the grid do not come from the use of a shorter time-step.

A similar behavior is found for the imposed-SST and coupled climate simulations shown here (Fig. 3): a tendency of the jets to move toward the poles when increasing the resolution, with a stronger impact when refining the grid in latitude. The effect is not as systematic as in the idealized dynamical simulations of Guemas and Codron (2011), which may reflect additional effects due to the complexity of the climate system.

In order to understand how the grid refinement impacts the SSTs, i.e. both the increase of the mean temperature and reduction of the latitudinal contrasts, we start by analyzing the change in thermodynamical variables and energy budget in the imposed-SST simulations.

\subsection{Thermodynamical variables in the imposed-SST simulations}

The changes in zonal winds shown in Fig. 4 are accompanied by systematic changes in the temperature and humidity fields.

The mid-latitude tropopause (close to $200 \mathrm{hPa}$ ) moistens when refining the horizontal grid, and becomes too moist when compared to ERA-Interim for the finest grids. The tropopause cold bias of the mid to high latitudes also increases. These two trends are probably related to each other since the cooling to space, a dominant term of the radiative balance at this level, is strongly affected by humidity as already discussed by Hourdin et al. (2006). Overall, the mid-latitude tropopause is thus too high for the finest grids explored.

The systematic dry bias of the tropical boundary layer top $(900 \mathrm{hPa})$ is a direct consequence of an underestimated moisture vertical transport by the eddy-diffusion parameterization used in LMDZ4. It is therefore not affected by the changes in horizontal resolution. 

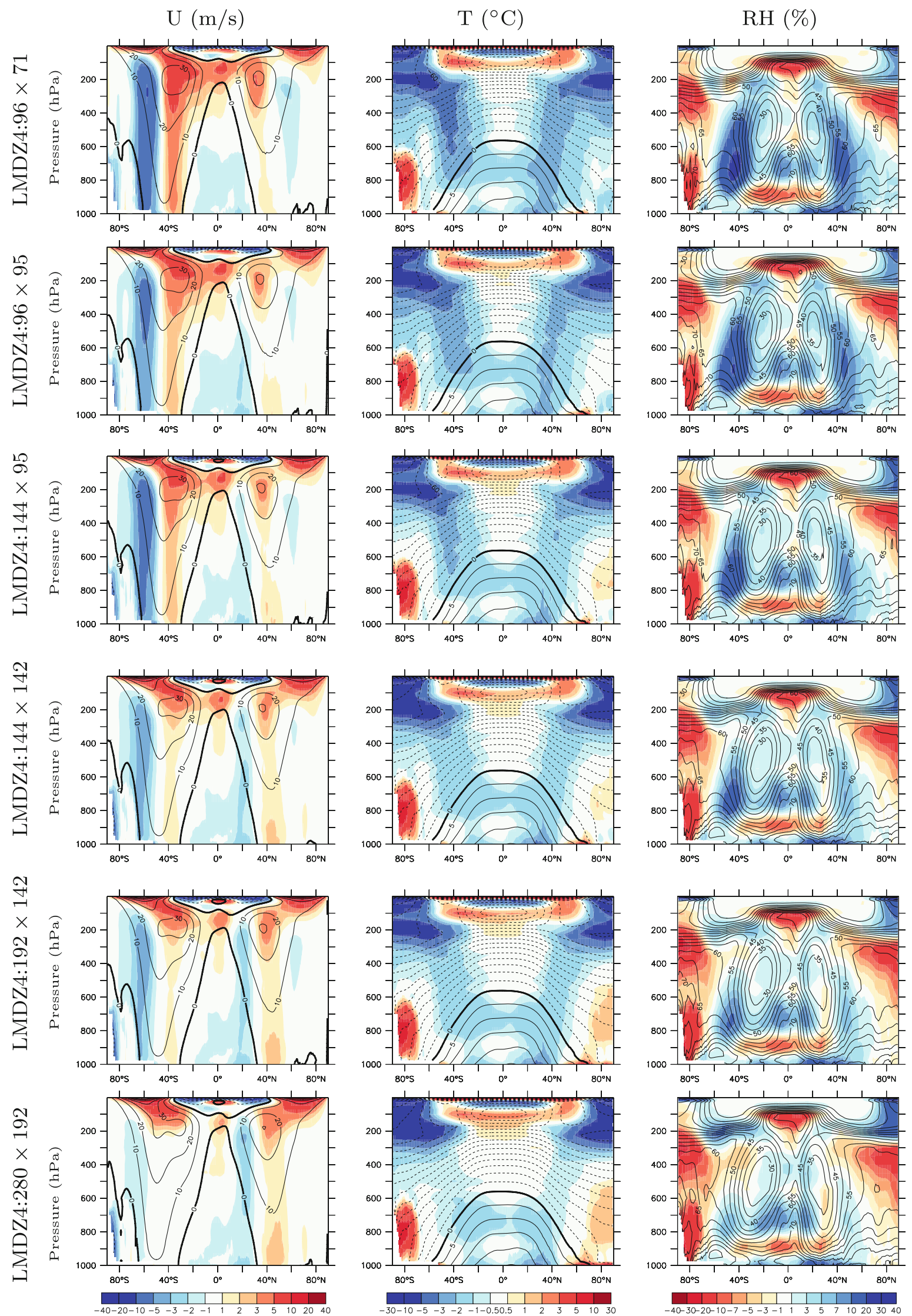
4Fig. 4 Ten-year average of the mean meridional structure of the zonal wind $(\mathrm{m} / \mathrm{s}$, left $)$, temperature $\left({ }^{\circ} \mathrm{C}\right.$, middle $)$ and relative humidity $(\%$, right) for the various imposed-SST simulations with LMDZ4 (L19). The contours correspond to the simulations and the colors to the difference (bias) with ERAinterim re-analayzes

Grid refinement leads to a systematic decrease of the wet and cold bias of the mid-latitude troposphere. This decrease of relative humidity is not just a consequence of the warmer temperature since the specific humidity is reduced as well, as illustrated in Fig. 5 a and b that show differences between the $96 \times 71$ and $144 \times 142$ grids. These changes can be interpreted as a shift toward the poles of the dry anticyclonic regions of the sub-tropics, as seen from the coincidence of the location of the maximum drying with that of the maximum latitudinal gradient of relative humidity (Fig. 5b).

The impact of the poleward displacement of the jet and of the Hadley-cell boundary is also apparent in the water budget. The difference of integrated meridional transport of moisture between the $144 \times 142$ and $96 \times 71$ resolutions is shown on Fig. 6 a (the transport of $L q$ is shown here where $L$ is the specific latent heat and $q$ the specific humidity). The Hadley circulation transports water toward the equator (more water being transported in the lower branch of the cell), while the Ferrel Cell and mid-latitude eddies transport moisture toward the pole. A wider Hadley cell will thus increase the equatorward transport near the latitudinal edge of the cell, while the displacement of the mid-latitude eddies will increase poleward transport in higher latitudes. The differential transport with increased resolution is therefore systematically away from the midlatitudes $\left(40^{\circ} \mathrm{N}\right.$ and $\left.40^{\circ} \mathrm{S}\right)$ towards the equator and poles. As a consequence, precipitation is reduced in the midlatitudes (Fig. 6 b), even though the evaporation increases weakly because of the drier atmosphere.

\subsection{Energy budget in the imposed-SST simulations}

The changes in relative humidity illustrated in Fig. $5 \mathrm{~b}$ between resolutions $96 \times 71$ and $144 \times 142$ coincide with large changes in cloud fraction (Fig. 5c). Specifically, the cloud fraction exhibits a significant decrease near $40^{\circ}$ latitude in both hemispheres, and a systematic increase at the tropopause.

The changes in clouds are associated with pronounced changes in the Top-of-Atmosphere (TOA) radiative budget (Fig. 6d). The short-wave (SW) Cloud-Radiative-Forcing (CRF), defined as the difference of the TOA SW radiation between all-sky and clear-sky conditions, is strongly increased in the mid-latitudes, as a consequence of the decrease of the fractional coverage of low and mid-level clouds. For long-wave (LW) radiation, the effect of clouds and the modification of clear-sky radiation partially cancel (a) Specific humidity : $\Delta q / q(\%)$

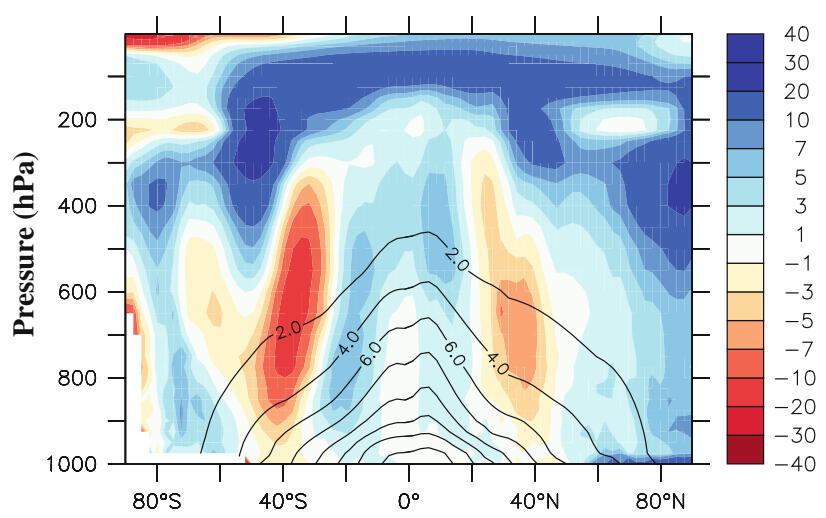

(b) Relative humiditiy : $\Delta \mathrm{RH}(\%)$

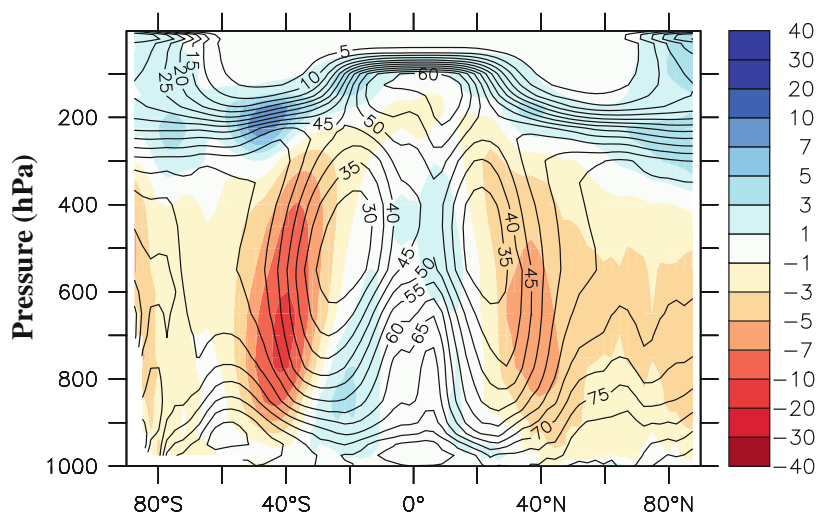

(c) Cloud cover : $\Delta \mathrm{f}(\%)$

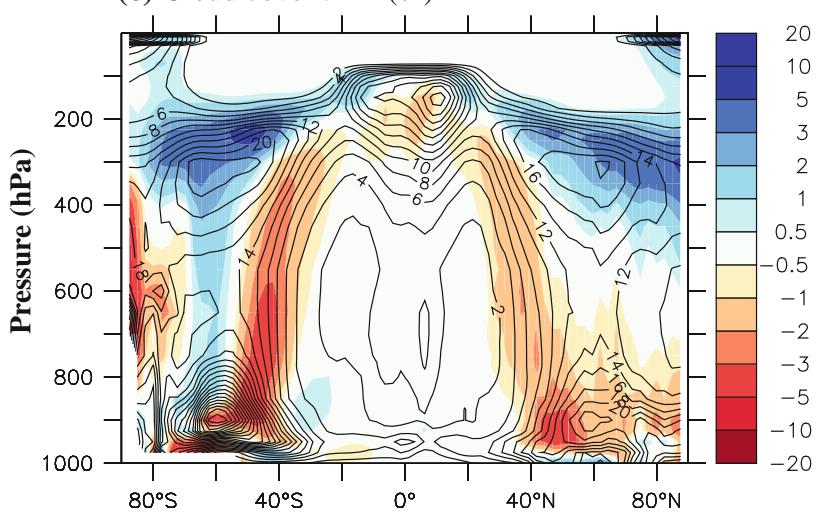

Fig. 5 Zonal mean change of the latitude-pressure distribution of moisture and clouds in LMDZ4 imposed-SST simulations associated with grid refinement from $96 \times 71$ to $144 \times 142$ : a relative difference in specific humidity (\%), b difference in relative humidity (\%) and $\mathbf{c}$ difference in cloud fraction (\%). The differences are in color while the contours correspond to the mean value of the $144 \times 142$ simulation (resp. in $\mathrm{g} / \mathrm{kg}, \%$ and $\%$ )

each other. The change in SW CRF does not affect significantly the atmospheric budget (red curve in Fig. 6c), since the increase of down-welling SW radiation at surface (red curve in Fig. 6e) is very close to that at TOA. Conversely, the decrease in low-level cloud cover and near surface humidity in the mid latitudes reduces the LW 
(a) Heat transp. $($ Etot $=\mathrm{Ed}+\mathrm{Lq}, \mathrm{Edry}=\mathrm{CpT}+\mathrm{gz})$
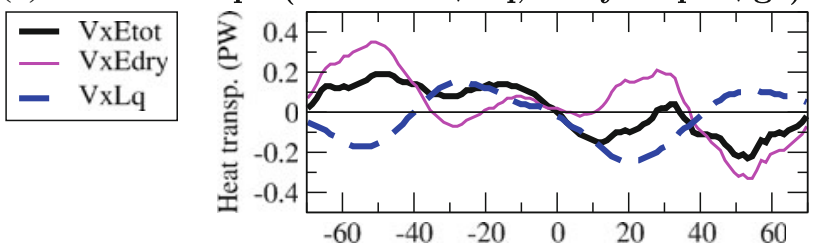

(b) Water budget
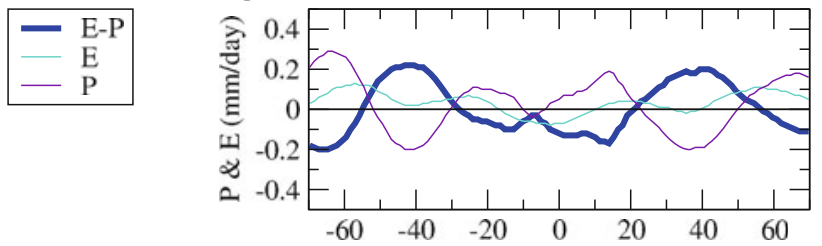

(c) Atmospheric budget (TOA - surface fluxes)
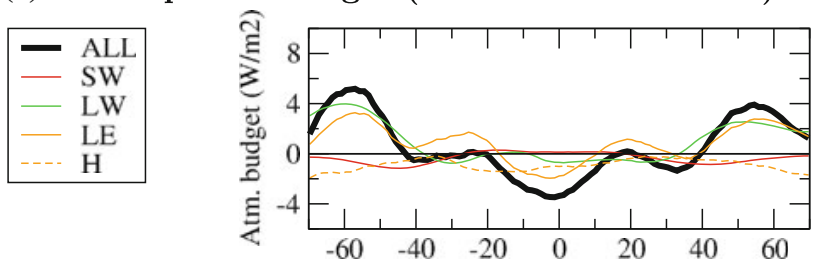

(d) TOA fluxes
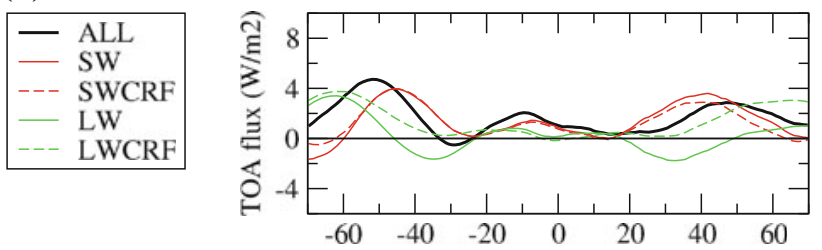

(e) Surface fluxes

\begin{tabular}{|ll|}
-- & ALL \\
- & SW \\
- & LW \\
-- & LWdown \\
- & Turbulent \\
--- & Tsol \\
\hline
\end{tabular}

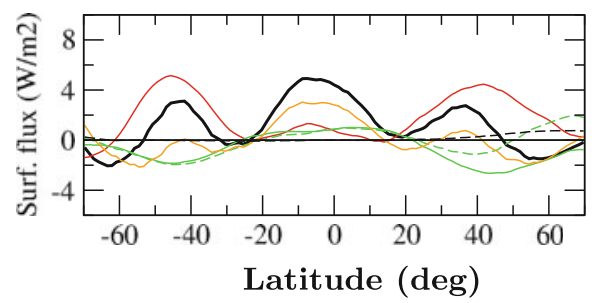

Fig. 6 Change in atmospheric transport, water and energy budget between the $96 \times 71$ LMDZ4 imposed SST simulation and the $144 \times 142$ configuration : a change in meridional energy transport (in PW), the moist static energy Etot being decomposed into its dry component $C_{p} T+g z$ and latent heat $L q$; b change in evaporation $(E)$, precipitation $(P)$ and water budget $(E-P)$; $\mathbf{c}$ change in atmospheric budget, difference between the TOA and surface (downward) fluxes, separating the contribution of SW and LW radiation, and the latent $(L E)$ and sensible $(H)$ heat flux at surface; d TOA fluxes, for LW and SW radiation together with the corresponding CRF; e surface downward fluxes. For the LW radiation, we show in green both the net radiation (full line) and down-welling radiation (dashed). The changes in turbulent flux $-(H+L E)$ and mean surface temperature are also shown in (e) (orange and dashed black curves respectively). In $\mathbf{c}$, $\mathbf{d}$ and e, ALL means the sum of the LW, SW and turbulent contributions. Note that a 20 -degree area conserving running mean is applied in latitude to all the fields in order to remove the numerical noise that results from differences computed between two different grids

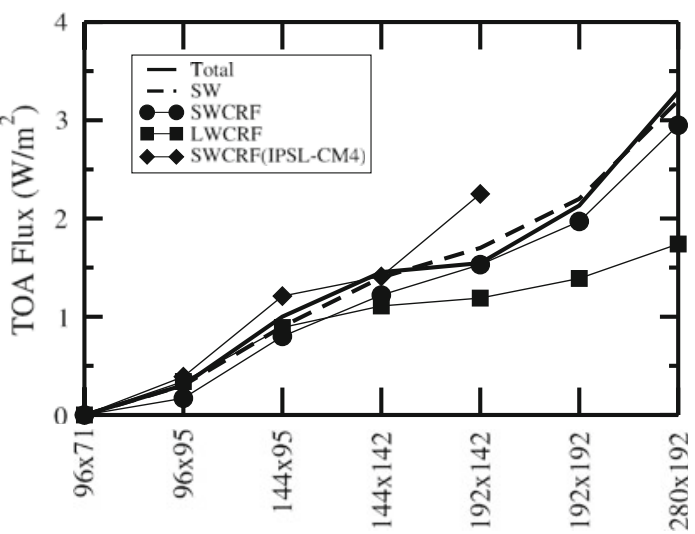

Fig. 7 Impact of the grid resolution on the top-of-atmosphere (TOA) fluxes $\left(\mathrm{W} / \mathrm{m}^{2}\right)$ in the imposed-SST LMDZ4 simulations. The total $(\mathrm{LW}+\mathrm{SW})$ net radiation (full curve) together with the SW component (dashed), SW CRF (circles) and LW CRF (squares) are shown. Results from the $96 \times 71$ simulation are subtracted. The SW CRF of the coupled IPSL-CM4 simulations (diamonds) is also shown for comparison. All the diagnostics correspond to 10-year means

radiation of the atmosphere toward the surface (green dashed curve in Fig. 6e). The change of net LW radiation (full green curve) is almost identical to the change in downweling LW radiation except in the northern mid and high latitudes where continental surfaces respond to the increased surface incoming SW radiation.

The sensible heat flux is reduced rather systematically by about $1 \mathrm{~W} / \mathrm{m}^{2}$ due to the warmer atmosphere. The latent heat is reinforced in the mid latitudes but with a local minimum at $40^{\circ}$ latitude. All together, the atmosphere is heated by diabatic processes in the mid-latitudes more than in the tropics, which induces a reduction of the total latitudinal energy transport (black curve in Fig. 6a). This decrease is however weak, with a partial compensation between the transport of $L q$ and that of the dry static energy $C_{p} T+g z$.

In the imposed-SST LMDZ4 simulations, the global value of the total longwave plus shortwave $(\mathrm{LW}+\mathrm{SW})$ radiation at TOA (full curve in Fig. 7) systematically increases with grid refinement. It changes by $+3 \mathrm{~W} / \mathrm{m}^{2}$ (a gain for the climate system) when going from the coarsest to the finest grid. For the global average, this additional heat for the climate system can be entirely explained by the change in SW CRF, associated with a reduction of the averaged low-level cloud cover from almost $27 \%$ for the $96 \times 71$ grid to less than $24 \%$ for the finest $280 \times 192$ grid. The LW CRF also increases with grid refinement but is compensated by a decrease of the clear-sky Outgoing Longwave Radiation (OLR, not shown), so that the total OLR is almost independent of grid resolution (as evidenced by the fact that the total and SW radiation almost coincide in Fig. 7). 
We detail below the specific modifications of the SW CRF that result from a grid refinement in either longitude or latitude. Refining the grid in latitude induces a maximum of SW CRF increase in the mid-latitudes (red curve in Fig. 8a) which may be explained by the latitudinal shift of the jets: the jets being closer to the pole in the finest grids, the region of strong (negative) SW CRF associated with the storm-tracks is shifted towards latitudes where the insolation is weaker, resulting in a weaker SW CRF and also in a weaker low-level cloud cover because of the wider meridional extent of the trades. Consistently, the grid refinement in latitude has a clear effect on the meridional water transport with a systematically increased transport away from the mid-latitudes, towards both the poles and the equator (red curves in Fig. 8b).

When increasing the resolution in longitude, the effect on the SW CRF is stronger in the tropics (blue curves in Fig. 8a), and is less clearly related to a change in the meridional moisture transport. This can be related to changes in the PDF (Probability Density Function) of the $500 \mathrm{hPa}$ large-scale vertical velocity, $\omega_{500}$, that characterizes the large-scale tropical circulation (Bony et al. 2004). The change in tropical dynamics is particularly clear when increasing the resolution in longitude from $96 \times 95$ to $144 \times 95$ (full blue curve in Fig. 8c). The deep convective regimes $\left(\omega_{500}<-40 \mathrm{hPa} /\right.$ day $)$ associated with the inter tropical convergence zone (ITCZ) and the strong subsiding regimes $\left(\omega_{500}>30 \mathrm{hPa} /\right.$ day $)$ associated with strato-cumulus regions both have a strongly reduced occurrence, with a compensating increase in the weakly subsiding regimes. These two extreme regimes correspond to maximum cloud coverage and SW CRF, so their diminution could partly explain the reduction of the SW CRF in the tropics, which was particularly large for this change of resolution. When increasing the resolution in latitude from $96 \times 71$ to $96 \times 95$ (red curve in Fig, 8c) the change of the PDF is dominated by a transfer from the weakly subsiding to the weakly ascending regimes so that the ascending motions are globally reinforced on the domain retained for analysis (the ocean in the $30 \mathrm{~S}-30 \mathrm{~N}$ latitude band). This increased ascent is compensated by subsidence in the extra-tropics. These changes are accompanied in this particular case by a slight decrease of the SW CRF in the tropics (Fig. 8). The effects of resolution changes are weaker when exploring finer resolutions, in terms of both PDF and SW CRF changes.

\subsection{Impact on SST in the coupled experiments}

The changes with resolution of the global SW CRF are quite similar in the coupled (Fig. 9) and imposed-SST simulations (the SW CRF of the coupled simulations is duplicated in Fig. 7 for comparison). The latitudinal distribution of these changes of SW CRF are also quite similar, as can be seen for
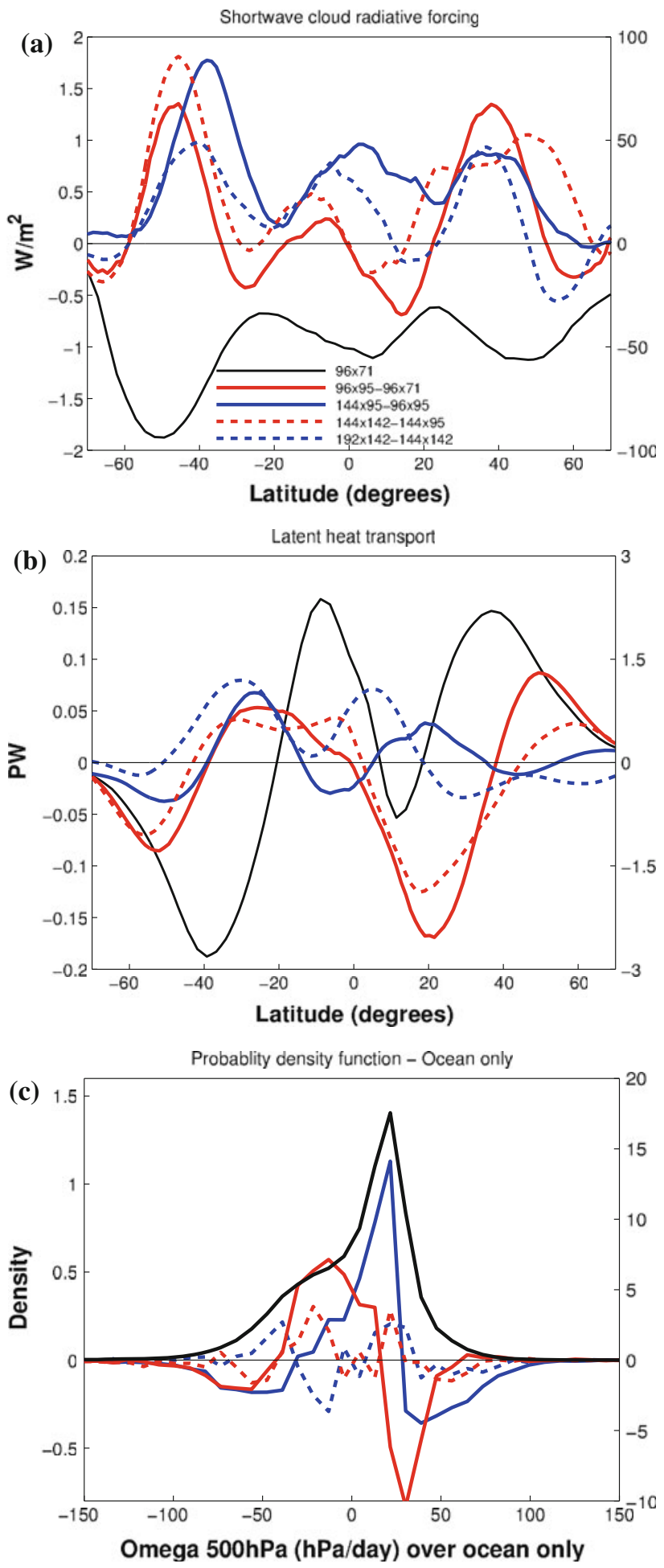

Fig. 8 Impact of grid refinement on: a the latitudinal distribution of the SW CRF, $\mathbf{b}$ the meridional transport of latent heat $(L q)$, and $\mathbf{c}$ the PDF (Probability Density Function) of the $500 \mathrm{hPa}$ large-scale vertical velocity $\omega_{500}$ in the Tropics $(30 \mathrm{~S}-30 \mathrm{~N})$. The red (respectively blue) curves show the difference between pairs of imposed-SST experiments with consecutive grid refinement in latitude (respectively in longitude). Scales are on the left vertical axis. For each graph, the black curve corresponds to the simulation with the $96 \times 71$ grid. The corresponding values are on the right vertical axis 


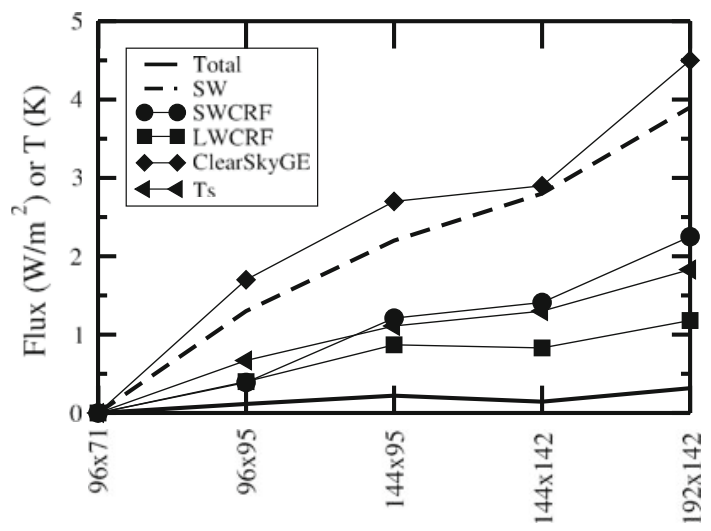

Fig. 9 Impact of the grid resolution on the TOA fluxes $\left(\mathrm{W} / \mathrm{m}^{2}\right)$ and global-mean surface temperature $T_{s}$ (triangles, in $\mathrm{K}$ ) in the coupled atmosphere-ocean IPSL-CM4 simulations. Results from the $96 \times 71$ simulation are subtracted. The total $(\mathrm{LW}+\mathrm{SW})$ net radiation (full curve) together with the SW component (dashed), SW CRF (circles), LW CRF (squares), clear sky greenhouse term (diamonds) are shown. All the diagnostics correspond to 10 -year means

resolutions $96 \times 71$ and $144 \times 142$ by comparing the dashed red curves in Figs. 6d and 10d.

In the coupled IPSL-CM4 simulations, the imbalance of the TOA radiative budget associated with grid refinement, coming from the change in SW CRF, acts as an initial forcing and induces a warming of the global surface temperature until a new equilibrium is reached. After 90 years, the total net flux in the simulations shown here is close to zero, as expected for an equilibrated coupled simulation. The total flux is of $-0.4 \mathrm{~W} / \mathrm{m}^{2}$ for the $96 \times 71$ grid. The other resolutions have a total balance less negative by a fraction of a W/m $/ \mathrm{m}^{2}$ (full curve in Fig. 9), indicating that the various simulations are not too far from radiative equilibrium. Note that there is an energy leakage of the order of $0.2 \mathrm{~W} / \mathrm{m}^{2}$ in all the coupled atmosphere-ocean simulations presented here (see Dufresne et al., this issue).

The direct forcing induced by the SW CRF change on the temperature is then amplified by classical climate feedbacks resulting from the surface temperature increase. This can be illustrated from the comparison of the $96 \times 71$ and $192 \times 142$ simulations, i.e. focusing on the values associated with grid $192 \times 142$ on the x-axis in Figs. 7 and 9. Simulations with imposed SST show that the initial SW CRF between the two resolution is $1.5 \mathrm{~W} / \mathrm{m}^{2}$ (circles in Fig. 7). The SW-CRF is reinforced by about $0.7 \mathrm{~W} / 2$ in the coupled experiments (positive feedback, as seen by comparing circles and diamonds in Fig. 7). The difference between the absorbed solar radiation (SW) and the SW CRF in Fig. 9 reflects a positive feedback from the surface albedo (of about $1.7 \mathrm{~W} / \mathrm{m}^{2}$ ), resulting from a decrease in snow and ice cover. Between the forced and coupled simulation the role of clouds on longwave radiation remains comparable (squares in Figs. 7 and 9). The change in TOA SW radiation is around $4 \mathrm{~W} / \mathrm{m}^{2}$ and so is the change in OLR in the coupled simulations.

The change in LW emission by the surface, $\sigma T_{s}^{4}$, can be formally decomposed as the sum of the change in OLR and in Greenhouse Effect (GE) term (Raval and Ramanathan 1989)

$G E=\sigma T_{s}^{4}-O L R$

with a strong contribution of the clear-sky GE (diamonds in Fig. 9) associated with water vapor and lapse rate feedbacks. Finally, a temperature increase of $1.8 \mathrm{~K}$ is obtained for an initial SW CRF of $1.5 \mathrm{~K}$ in the imposed-SST simulations. The sensitivity close to $1.2 \mathrm{~K}$ per W/m² ${ }^{2}$, obtained here from a change in horizontal grid, is comparable to that obtained in climate change simulations with the IPSL-CM4 model.

In terms of modification of the latitudinal structures, the results also follow what was observed in imposed-SSTs simulations, but for the above mentioned feedbacks. As was the case in the imposed-SSTs simulations, the atmospheric transport tends to dry the mid latitudes (Fig. 10a and b). The TOA SW CRF shows, similarly, a maximum increase in the mid latitudes. The surface albedo feedback is seen by the fact that the change in total TOA SW radiation (full red curve in Fig. 10d) in the high latitudes is somewhat larger than the CRF (dashed) while the two curves were almost superimposed in the imposed SST simulations (Fig. 6d). The coupled atmosphere-ocean system tends to re-adjust to this SW forcing, so that, at the end, the increased OLR in the mid latitudes almost compensates for the increased SW radiation (green and red full curves of Fig. 10d). The surface temperature increase (black dashed curve in Fig. 10e) is somewhat larger in the mid- and high-latitudes than at the equator so that the turbulent fluxes (latent + sensible) tend to increase specifically at those latitudes (an heating for the atmosphere). The new equilibrium in the coupled model also results in a reduction of the atmospheric equator to pole heat transport (Fig. 10a). However the magnitude is smaller than in the atmosphere alone simulations, mainly because the warmer SSTs lead to enhanced evaporation with resolution that counterbalances the surface net radiation, which smoothes the changes in the atmospheric equator to pole energy budget compared to the imposed-SST simulations.

\subsection{Oceanic transport}

The poleward shift of the jets has a positive impact on the ocean gyre circulation in the north Atlantic (illustrations not shown). The warm and saltier water from the tropics are advected further north in the Atlantic, which reinforces deep water formation and the northward heat transport by the ocean circulation by up to $0.15 \mathrm{PW}$ at $30^{\circ} \mathrm{N}$ between the coarsest $(96 \times 71)$ and finest $(144 \times 142)$ grid. The 
(a) Heat transp. $($ Etot $=$ Edry + Lq, Edry $=\mathrm{CpT}+\mathrm{gz})$
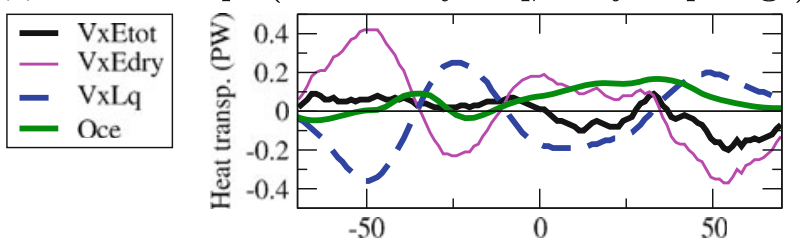

(b) Water budget
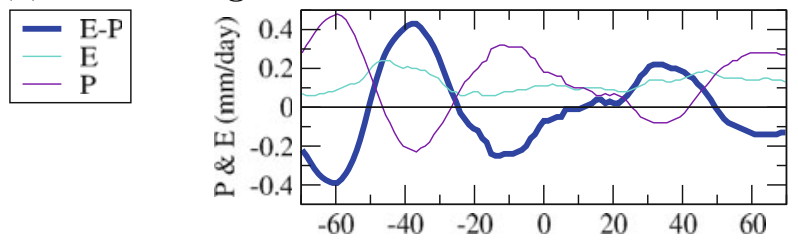

(c) Atmospheric budget (TOA - surface fluxes)

\begin{tabular}{|ll|}
- & ALL \\
- & SW \\
- & LW \\
- & LE \\
$-\cdots$ & H \\
\hline
\end{tabular}

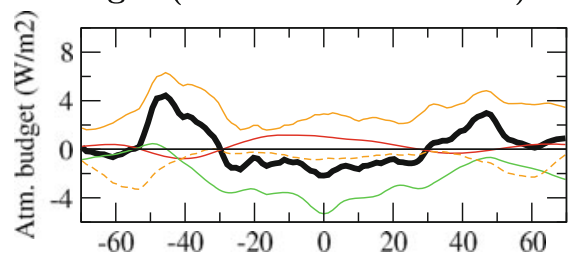

(d) TOA fluxes
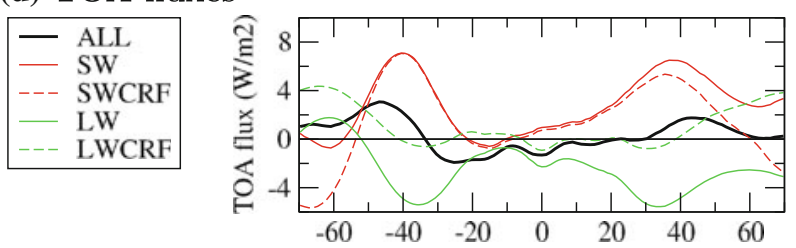

(e) Surface fluxes
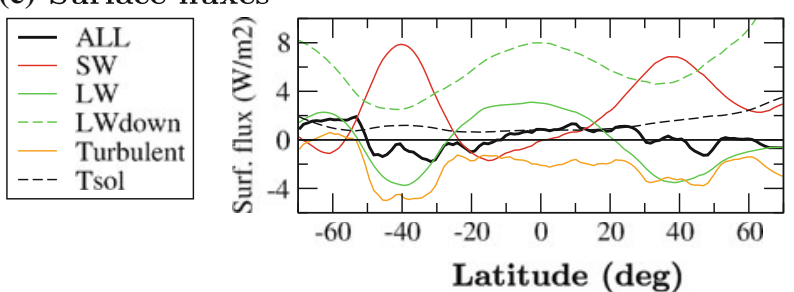

Fig. 10 Same as Fig. 6 but for the coupled IPSL-CM4 simulations. The change in oceanic heat transport is added on a (thick green curve)

processes involved are similar to the one discussed by Marti et al. (2010). The role of the Atlantic in the northern hemisphere is directly reflected on the changes in the global heat transport by the ocean circulation (thick green curve in Fig. 10a). These changes in the ocean circulation partially counteract the reduction of the heat transport by the atmospheric circulation discussed above. However the changes in the atmosphere of about $0.2 \mathrm{PW}$ between the coarsest and finest grid considered here are larger than those of the ocean so that the sum of the heat transport by the ocean and the atmosphere reduces with resolution, reflecting the dominant role of the readjustments of temperature, humidity and clouds in the atmospheric column on the new equilibrium.

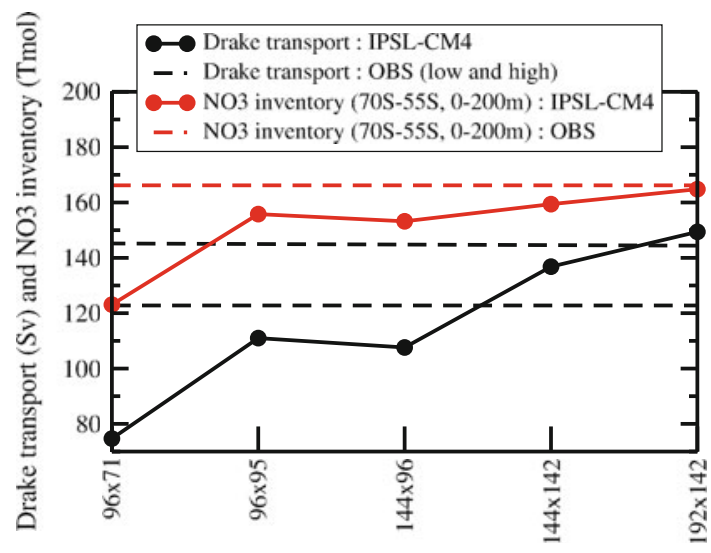

Fig. 11 Volume transport through Drake Passage (between the southern tip of America and Antarctica), in black, and nitrate inventories in the Southern Ocean (90S-55S, 0-200m), in red. Circles are for the IPSL-CM4 simulations with various horizontal grids. Data (dashed lines) correspond to Cunningham et al. (2003) for the Drake transport and Conkright et al. (2002) for nitrate inventories. Note that the PISCES biogeochemical model has been run offline for the results shown here, with the same ocean grid configuration for all the atmospheric grids

The southward shift and intensification of the westerlies associated with the poleward shift of the atmospheric midlatitude jets with resolution increase the mass flux of the Antarctic Circumpolar Current, as shown by the volume transport through Drake Passage (Fig. 11). These changes have a positive impact on the representation of the nutrient fields in the ocean. It can be inferred from simulations performed with the PISCES biogeochemical model (Aumont and Bopp 2006) forced by the CM4 ocean circulation. We focus here on the first $200 \mathrm{~m}$ nitrate inventories in the Southern Ocean (south of $55^{\circ} \mathrm{S}$ ). These inventories are key in setting the Southern Ocean biological productivity, and also in determining the nutrient concentrations of the tropical oceans (Sarmiento et al. 2004). The suite of CM4 simulations clearly shows that changes in ocean transport and mixing due to the strengthening and poleward shift of the westerlies impact $\mathrm{NO}_{3}$ inventories (red curves in Fig. 11). The first $200 \mathrm{~m} \mathrm{NO}_{3}$ inventory increases from 123.1 Tmol to $164.8 \mathrm{Tmol}$ for an increase in atmospheric latitudinal resolution from 96 points $\left(1.9^{\circ}\right)$ to 142 points $\left(1.3^{\circ}\right)$, in better agreement with observations. These results illustrate both the importance of atmospheric dynamics representation for the other components of the "Earth System" and the potential new constraint that new components can provide for model evaluation.

\subsection{Impact on precipitation}

One motivation to increase the horizontal resolution of the atmospheric models is the better representation of rainfall distribution, a key variable for impact studies. We show in 
Fig. 12 Annual mean rainfall (mm/day) in the GPCP (Global Precipitation Climatology Project, Huffman et al. 2001) observations and for the two extreme configurations explored with LMDZ4

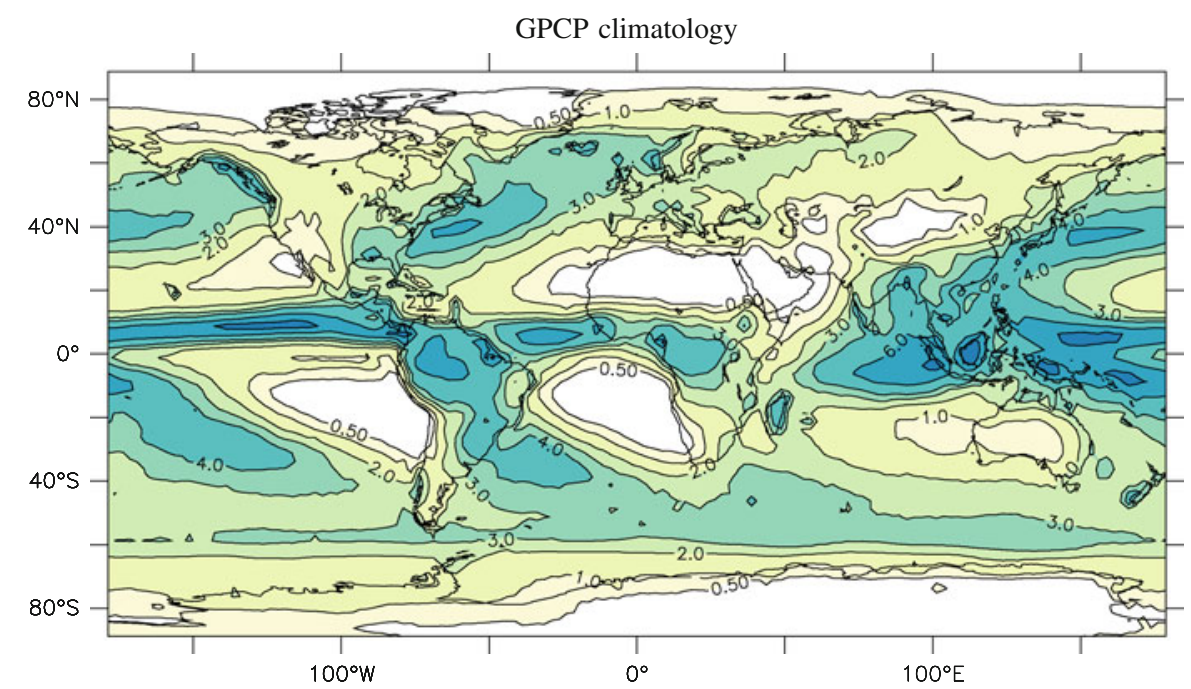

LMDZ4 - $96 \times 71$

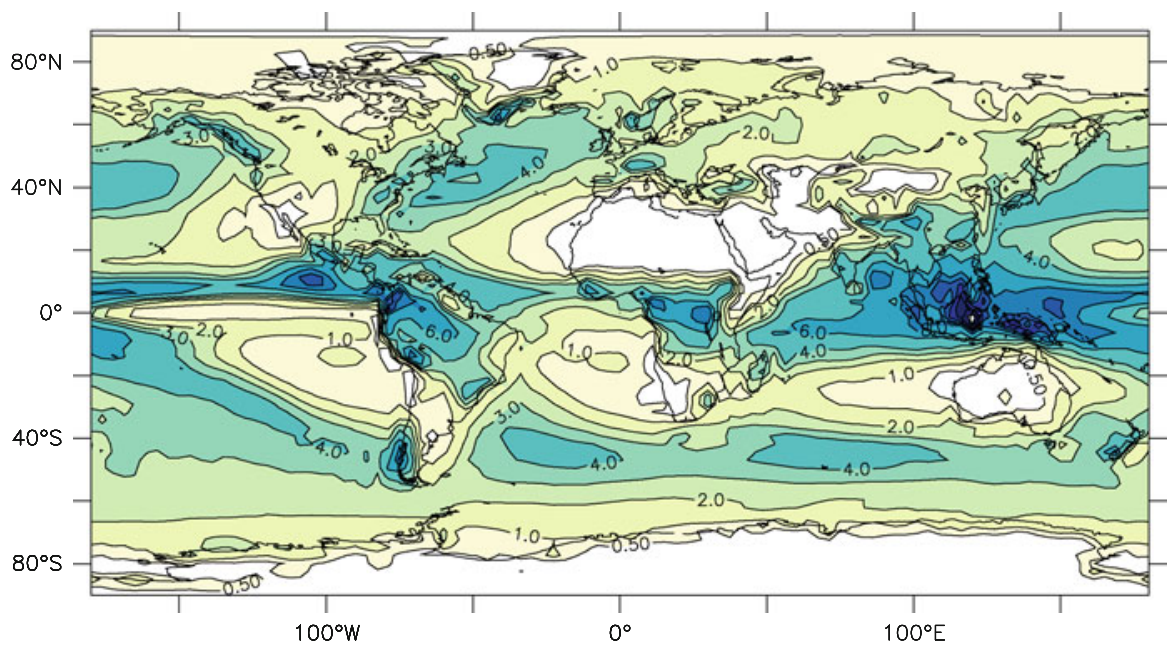

LMDZ4 - $280 \times 192$
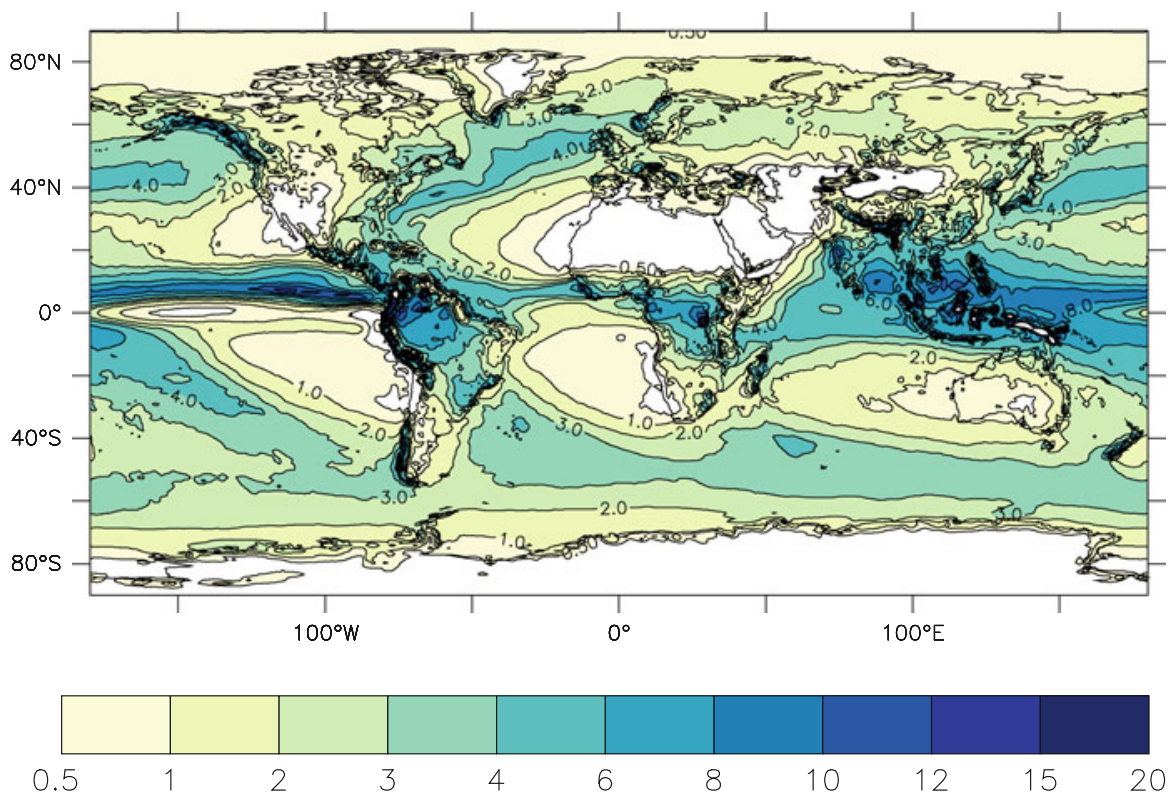


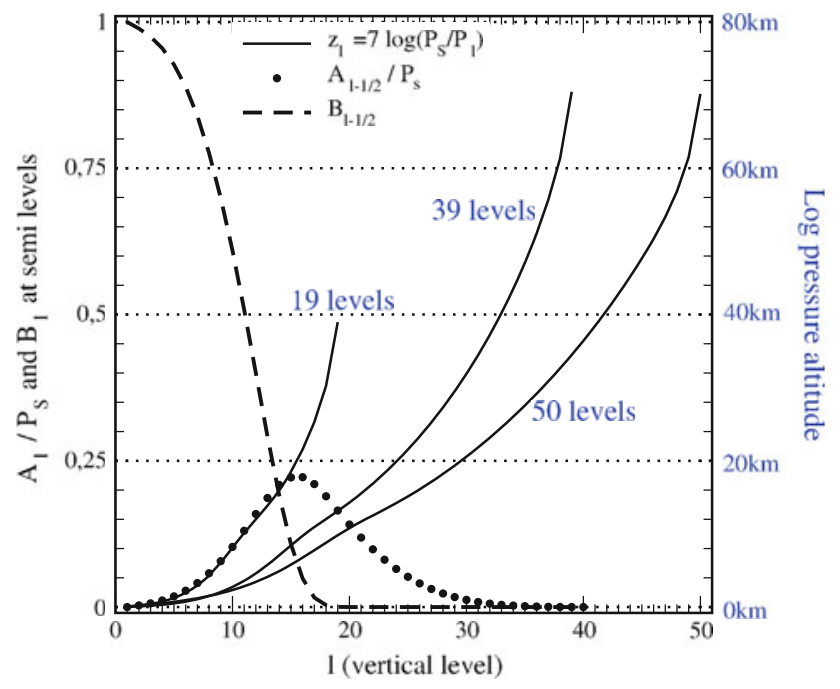

Fig. 13 Coefficients $A_{l}$ and $B_{l}$ defining the L39 vertical grid (dot and dashes line respectively). The thick lines are for the log pressure altitude in km: $z=7 \cdot \log \left(P_{s} / P_{l}\right)$. The values given correspond to the 2 versions used in this paper: the L19 vertical grid of LMDZ4 and the L39 grid of LMDZ5A. Also shown for comparison are the variation of $z$ with model level used in the standard L50 stratospheric version of LMDZ presented in Lott et al. (2005)

Fig. 12a a comparison of the annual-mean rainfall obtained for the coarsest $(96 \times 71)$ and finest $(280 \times 192)$ grids for the imposed-SST simulations. Despite a reduction by a factor 8 of the grid cells area, the differences are relatively weak. The northward extension of the West Africa monsoon rainfall at the southern edge of the Sahara desert, is for instance almost the same in the two versions (not far enough to the north for both). The tendency of the model to predict a double ITCZ structure in the East Pacific, with a too strong secondary zone of precipitation south of the equator, is also present in the two versions. The main differences come from a finer description of local rainfall patterns driven by orography, as over the Alps or the western Ghats (India).

\section{Extending the model to the stratosphere}

\subsection{The L39 vertical discretization}

During the preparation of the CMIP5 exercise, the vertical grid of LMDZ4, formerly based on a L19 (19 layers) discretization, was extended in the stratosphere using a L39 discretization as explained below. The model uses a classical hybrid $\sigma-P$ coordinate: the pressure $P_{l}$ in layer $l$ is defined as a function of surface pressure $P_{s}$ by $P_{l}=A_{l}$ $P_{s}+B_{l}$. The values of the $A_{l}$ and $B_{l}$ coefficients are chosen in such a way that the $A_{l} P_{s}$ part dominates near the surface (where $A_{l}$ reaches 1), so that the coordinate follows the surface topography (like the $\sigma$ coordinates), and $B_{l}$ dominates above several $\mathrm{km}$ of altitude, making the coordinate equivalent to a pressure coordinate there.

The $A_{l}$ and $B_{l}$ coefficients retained for the former L19 and the new L39 configurations are as shown in Fig. 13. The L39 discretization goes up to about the same altitude of $70 \mathrm{~km}$ as the stratospheric L50 version used in Lott et al. (2005), and much higher than the L19 version. With 15 levels above $20 \mathrm{~km}$, the resolution of the L39 configuration is sufficient to resolve the propagation of the mid-latitude waves into the stratosphere and their interaction with the zonal-mean flow as illustrated below. Sudden-stratospheric warmings are thus simulated, but not the Quasi-Biennial Oscillation in the tropics. Since the L39 version goes to the same height as the L50 version described in Lott et al. (2005), we use the same parameters for the orographic and non-orographic gravity waves.

\subsection{Representation of the stratospheric variability}

The L39 vertical resolution retained here is in practice sufficient to capture the planetary waves that control the polar vortex dynamics in the stratosphere (Charney and Drazin 1961). This is illustrated in Fig. 14 which shows, for coupled atmosphere-ocean simulations with the $96 \times 95$ atmospheric configuration, the amplitude of the first 3 stationary planetary waves that modulate the northern stratospheric polar vortex in January. These amplitudes are computed by expanding the geopotential altitude $Z$ in Fourier series,

$Z(\lambda, \phi, z, t)=\sum_{s} Z_{s}(s, \phi, z, t) e^{i s \lambda}$

where $\lambda, \phi$, and $z$ are the longitude, latitude and the $\log$ pressure altitude respectively, and by averaging the complex Fourier coefficients $Z_{s}$ over the days belonging to the 30 Januaries 1976-1995, yielding the temporal average $<Z_{s}>$. The amplitudes $\left\|<Z_{s}>\right\|=\sqrt{\left\langle Z_{s}><Z_{s}>^{*}\right.}$ of the first three planetary waves (Fig. 14) are comparable in the L39 version and in the reanalysis data. The level of realism is comparable with that of the L50 stratospheric version of LMDZ (see the Fig. 2 in Jourdain et al. 2008). The planetary waves in the L19 version are quite realistic in the troposphere, but are clearly underestimated in the lower stratosphere below $z=35 \mathrm{~km}$. This shows that a well-resolved stratosphere does not affect directly the planetary-scale waves in the troposphere, and that our L19 tropospheric model is damping adequately the waves near its top. Note that these results on the mean planetary waves remain essentially valid when looking at other months but also at the variability associated with each wave (as for instance also shown in the Fig. 2 in Jourdain et al. 2008). 

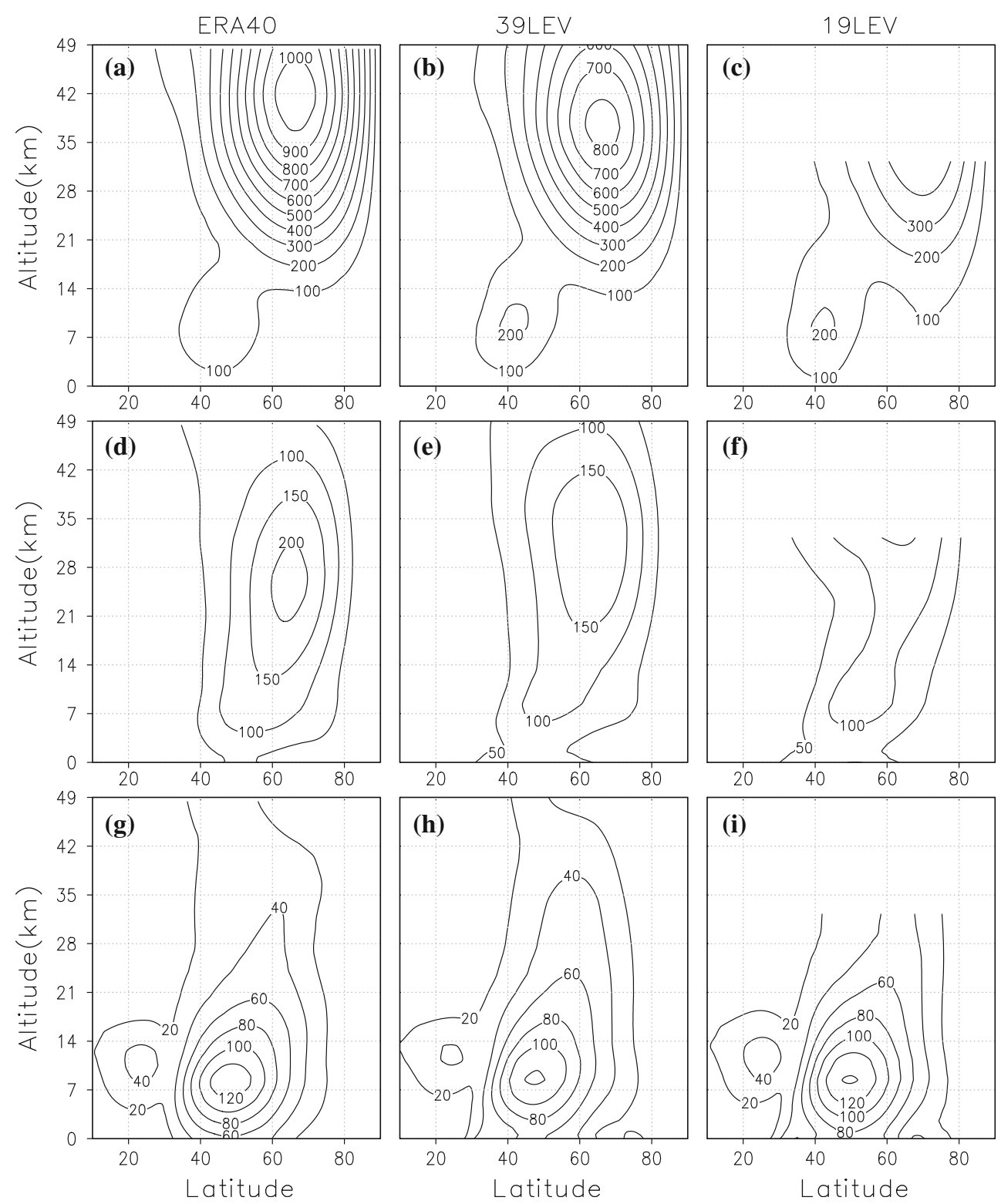

Fig. 14 Climatological amplitude of the first three dominant January planetary waves in the Northern Hemisphere. a-c wave $s=1$ from ERA40 and from L39 and L19 coupled simulations with the $96 \times 95$

To see whether the planetary waves are able to force stratospheric sudden warmings, we compare in Fig. 15 time-series of the zonal-mean temperature at $50 \mathrm{hPa}$ and $85^{\circ} \mathrm{N}$. We choose this altitude, which is significantly lower than the more conventional $10 \mathrm{hPa}$ level often used to diagnose the stratospheric warmings, because $10 \mathrm{hPa}$ is very close to the L19 model top (around $32 \mathrm{~km}$, see Fig. 13). Despite of this caveat, we see that the L19 version fails to simulate the right amount of polar temperature variability, whereas the $\mathrm{L} 39$ version is reasonably close to observations. This suggests that a realistic representation of the planetary waves in the upper stratosphere is necessary to represent sudden stratospheric warmings. In the L19 horizontal grid; d-f wave $s=2$ from ERA40, and from L39 and L19 simulations; g-i wave $s=3$ from ERA40, and from L39 and L19 simulations

version, the polar temperatures also present a cold bias of $10-20 \mathrm{~K}$ during the entire winter, and the average downward control related to the planetary waves breaking is not well represented, despite the fact that the planetary waves are quite realistic up to the L19 model top.

\subsection{Need for tuning}

Increasing the vertical resolution has a major impact on the TOA radiation budget in imposed-SST simulations, as shown in Table 2 and Fig. 16 that compare the L19 simulation with the simulation L39*, in which only the vertical resolution was increased without any specific tuning of the 

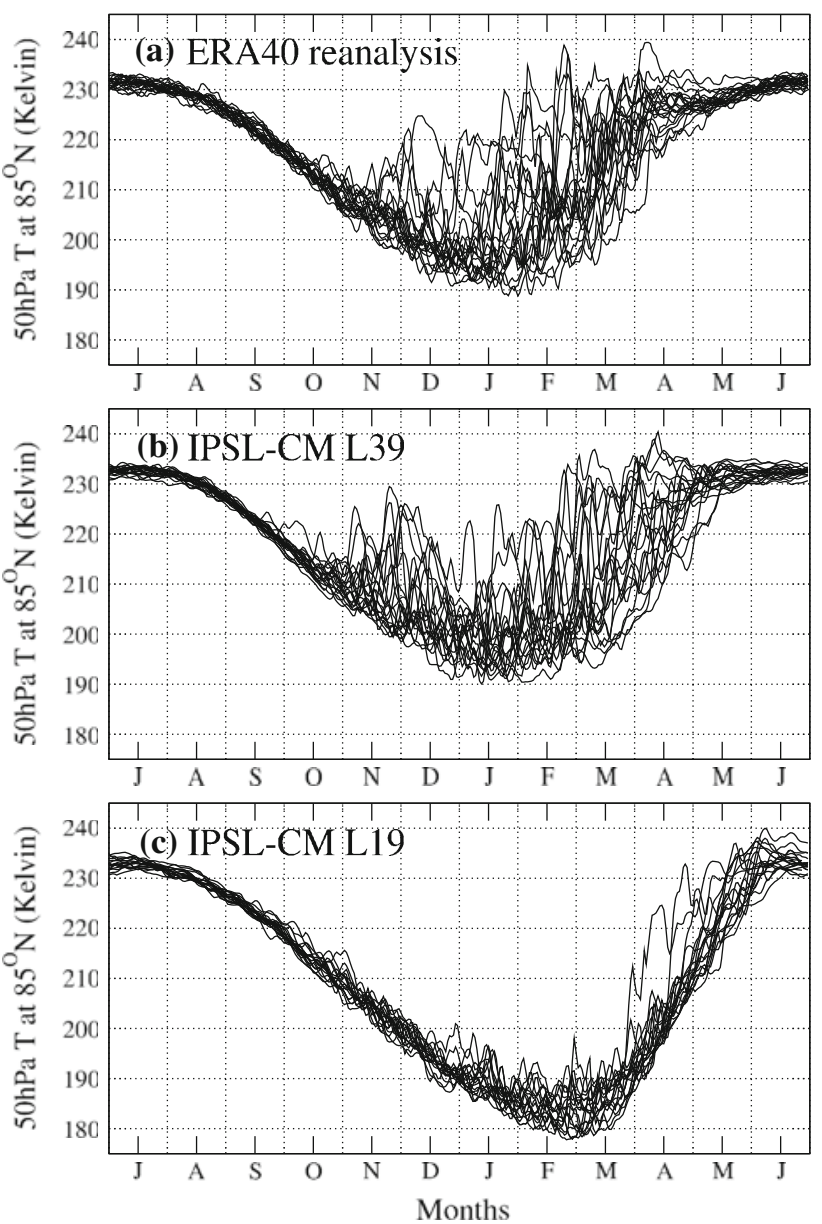

Fig. 15 Polar temperatures at $50 \mathrm{hPa}$ for 20 years (1976-1995). a ERA40 reanalysis, b L39 and c L19 IPSL-CM simulations

Table 2 Global values (in $\mathrm{W} / \mathrm{m}^{2}$ ) at TOA of the SW and LW CRF as well as of the total net radiation for imposed-SST simulations with LMDZ4-96 $\times 95$ for the L19 discretization and for the L39 discretization before (L39*) and after retuning of clouds parameters

\begin{tabular}{lllll}
\hline Simulation & SW CRF & LW CRF & Tot CRF & Total TOA \\
\hline $96 \times 95-L 19$ & -47.3 & 30.1 & -17.2 & -1.4 \\
$96 \times 95-L 39 *$ & -49.4 & 25.4 & -24.0 & -7.6 \\
$96 \times 95-L 39$ & -47.4 & 30.3 & -17.1 & -0.2 \\
\hline
\end{tabular}

model. The global net TOA atmospheric radiation decreases by about $6 \mathrm{~W} / \mathrm{m}^{2}$, with $2 \mathrm{~W} / \mathrm{m}^{2}$ coming from an increase of the (negative) $\mathrm{SW}-\mathrm{CRF}$ and $5 \mathrm{~W} / \mathrm{m}^{2}$ coming from a decrease of the (positive) LW-CRF. The clear-sky radiation is not strongly affected by the change of vertical resolution. The changes in CRF come from a decrease of the cloud cover in the upper troposphere and increase of boundary layer clouds as seen in Fig. 17.

A phase of tuning was thus required to re-equilibrate the TOA budget. The requirements on the accuracy of the TOA energy balance are much more stringent than the typical biases and approximations of climate models, in particular regarding cloud coverage and radiative properties. A modification of $1 \mathrm{~W} / \mathrm{m}^{2}$ of the TOA balance typically results in a change of $1 \mathrm{~K}$ of the global-mean surface temperature in a coupled model.

The tuning was done by considering a sub-set of the free parameters of the cloud parameterizations. Two parameters governing the upper-level clouds were modified. The maximum precipitation efficiency $\epsilon_{\mathrm{pr}, \max }$ of the Emanuel deep convection scheme, a critical and not well constrained parameter was changed from 0.99 to 0.999 . The fall velocity of the ice particles was divided by two by changing from 0.5 to 0.25 the value of a scaling factor $\gamma_{i w}$ introduced on purpose for model tuning in the formulation of the free fall velocity: $w_{i w}=\gamma_{i w} \times w_{0}, w_{0}=3.29$ $\left(\rho q_{i w}\right)^{0.16}$ being a characteristic free fall velocity (in $\mathrm{m} / \mathrm{s}$ ) of ice crystals given by Heymsfield and Donner (1990) where $\rho$ is the air density $\left(\mathrm{kg} / \mathrm{m}^{3}\right)$ and $q_{i w}$ the ice mixing ratio. The two changes compensate each other to some extent: a larger $\epsilon_{\mathrm{pr}, \max }$ results in a smaller detrainment of condensed water in the upper atmosphere while a smaller fall velocity reduces the main sink for total water. However, the first one mainly acts in the tropics, where the deep convection scheme is mostly active, while the second one has an impact at all latitudes. The specific choice made here results in a large increase of cloud cover (lower panel of Fig. 17) and humidity (not shown) in high latitudes, close to the tropopause level. This increase of high cloud cover has a clear signature in the LW CRF (green curve in the mid-upper panel of Fig. 16) in the imposed-SST simulations.

The last tuning parameter governs the conversion of cloud water to rainfall in the large-scale cloud scheme. Following Sundqvist (1978), the cloud liquid water (of mixing ratio $q_{l w}$ ) starts to precipitate in LMDZ5A above a critical value $c l w$ for condensed water, with a time constant for auto-conversion $\tau_{\text {convers }}=1,800 \mathrm{~s}$ so that

$\frac{d q_{l w}}{d t}=-\frac{q_{l w}}{\tau_{\text {convers }}}\left[1-\mathrm{e}^{-\left(q_{l w} / c l w\right)^{2}}\right]$

The critical value $c l w$ was changed from $0.26 \mathrm{~g} / \mathrm{kg}$ to $0.416 \mathrm{~g} / \mathrm{kg}$, within the typical range expected for cumulus and strato-cumulus clouds.

With the new tuning, L39 is close to the previous L19 version regarding the TOA gobal fluxes and CRF (Table 2) and the latitudinal distribution of the Net CRF (Fig. 16). However, this similarity of the net forcing is obtained in the tropics thanks to an error compensation between SW and LW CRFs which are both underestimated. The tuning of the original L19 version was probably better in that respect.

The retuning of the model has also a significant effect on the mean meridional structure of the meteorological fields. 

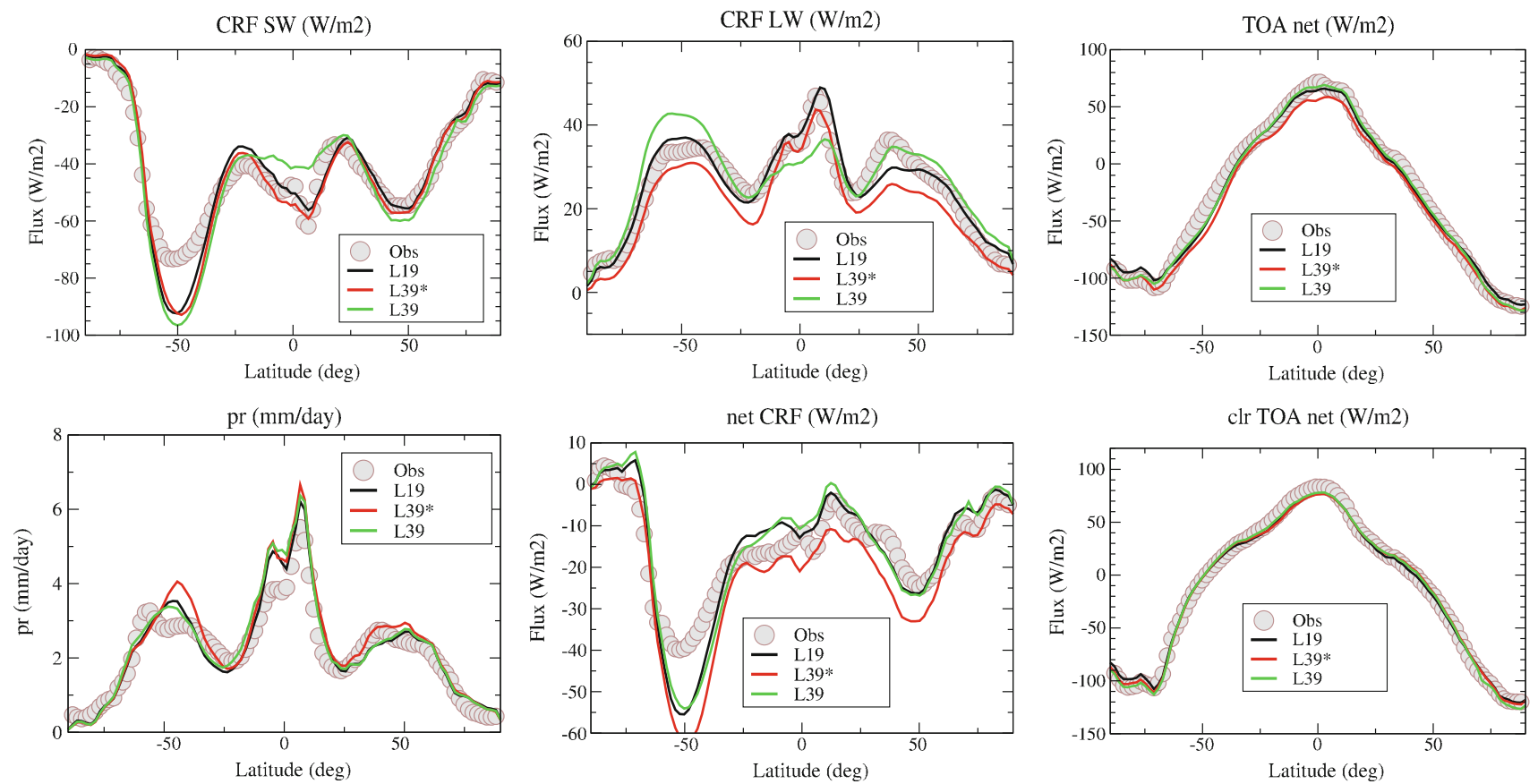

Fig. 16 Ten-year mean zonally averaged SW and LW CRF at TOA, net radiation, precipitation, net $\mathrm{CRF}$ and clear sky net radiation for imposed-SST simulations with the LMDZ4-96 × 95 configuration. Precipitation is in $\mathrm{mm} /$ day and fluxes in $\mathrm{W} / \mathrm{m}^{2}$. For radiative fluxes, observations correspond to the CERES Energy Balanced and Filled

We show in Fig. 18, in a latitude log-pressure framework, the mean biases in zonal wind, temperature and relative humidity in the L19, L39* (before retuning) and L39 (after retuning) versions. There is a significant improvement in the zonal wind structure in the stratosphere when going from L19 to L39*, which is partly reversed by the tuning. Also, the temperature biases around the tropopause in the mid-latitudes are a little bit stronger in L39 than in L39*. On the other hand, the retuning improves the location of the tropospheric jets, which is critical for the biases of the coupled model. This improvement is similar to the one obtained when using the $144 \times 142$ horizontal grid in the LMDZ4-L19 configuration (Fig. 4).

The retuning thus results in a compromise. On the one hand, the TOA fluxes and the stratospheric structure are degraded. On the other hand, the tropospheric circulation is improved, allowing the use of a coarser model resolution. The L39 vertical resolution, with the tuning described here, was retained for the LMDZ5A version of LMDZ used in IPSL-CM5A for the CMIP5 simulations.

\section{IPSL-CM5A versus IPSL-CM4}

The LMDZ4 configuration which was used in IPSL-CM4 for the CMIP3 simulations had the coarsest grid explored in the previous sections $(96 \times 71-\mathrm{L} 19)$. Apart from some
(EBAF) dataset, developed to remove the inconsistency between average global net TOA flux and heat storage in the Earth-atmosphere system (Loeb et al. 2009). We use GPCP (Huffman et al. 2001) for rainfall observations

minor bug fixes and optimization for parallel computing, the main differences between LMDZ versions used in the previous IPSL-CM4 version and the IPSL-CM5A one used for CMIP5 concern the grid configuration. The L39 discretization described above is retained in LMDZ5A with two horizontal grid configurations: a low resolution (LR) with $96 \times 95$ points $\left(3.75^{\circ} \times 1.9^{\circ}\right)$ and a medium resolution (MR) with $144 \times 142$ points $\left(2.5^{\circ} \times 1.25^{\circ}\right)$.

The Nemo ocean circulation model was also upgraded from OPA8 to OPA9 configuration, with in particular an improved vertical mixing scheme and an improved representation of solar absorption, as well as the Orchidee surface-vegetation-atmosphere transfer and dynamic vegetation model (de Rosnay et al. 2002; Krinner et al. 2005), with modified root profiles and an interactive Leaf Area Index computation. Those changes are detailed by Dufresne et al. (this issue). In this section, we document and discuss the effect of these configuration changes on the atmospheric component on the simulated coupled atmosphere-ocean climate and on its sensitivity to greenhouse gases concentration.

\subsection{Mean climate}

We compare here the control simulation with the IPSLCM4 $96 \times 71$ standard configuration (run with present-day forcing for greenhouse gases, solar constant, and aerosols) 

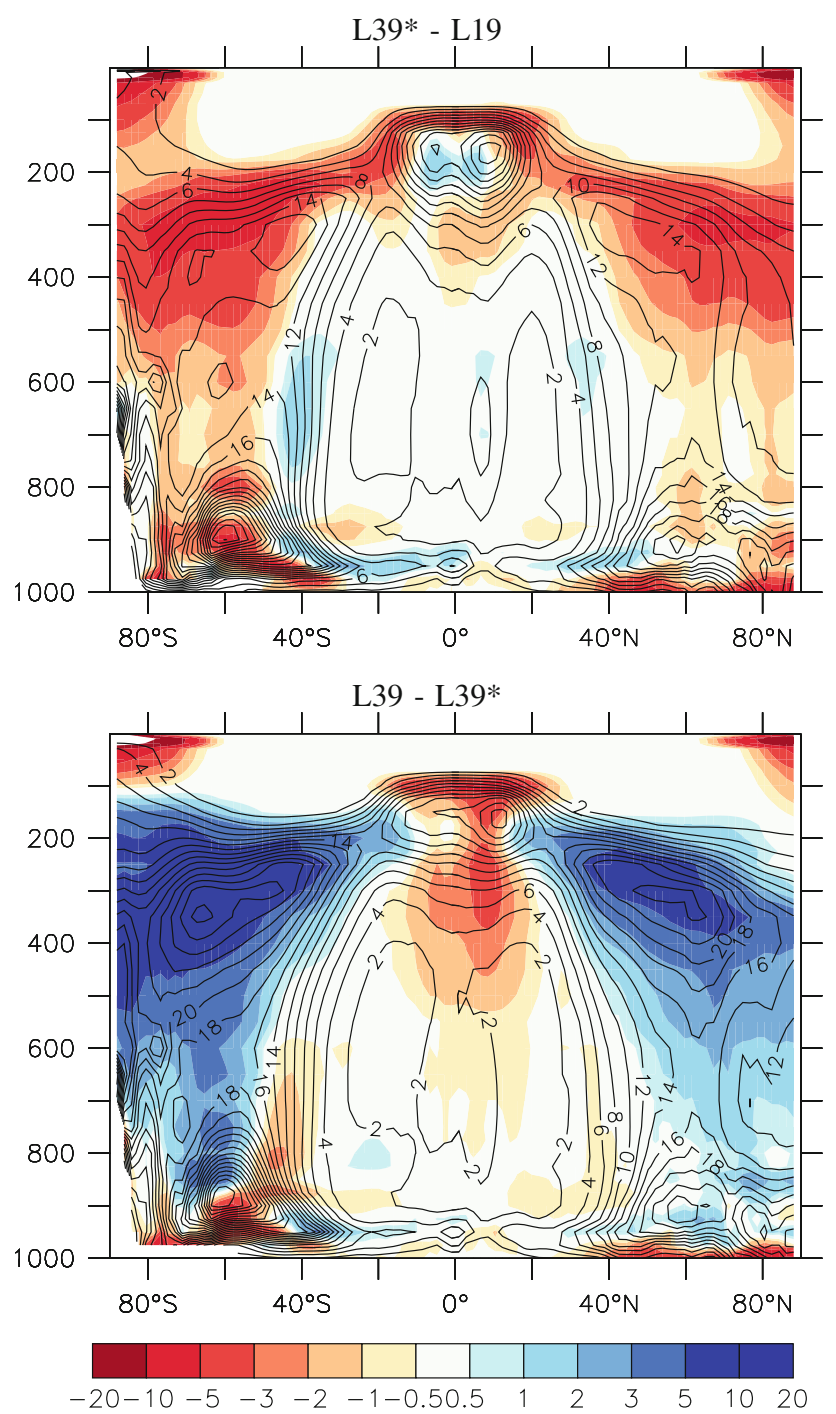

Fig. 17 Difference in cloud cover (annual and zonal mean) between (upper panel) the L19 and L39* simulation (with no tuning) and (lower panel) between the L39* and re-tuned L39 version. The LMDZ4-96 × 95 configuration is used

with the IPSL-CM5A-LR and MR "historical" simulations for 1990-1999. Those simulations start from the preindustrial control simulation. The evolution of the forcing from 1850 to 2000 is imposed as explained by Dufresne et al. (this issue). Those simulations are designed to be as close as possible to the observed climate. The LR configuration was considered too cold and thus a somewhat modified tuning was retained for the MR. This was done by subtracting a constant value of $1 \%$ from the ocean albedo.

The changes in the coupled simulations (Fig. 19) reflect for a large part the ones observed in the imposed-SST simulations (Fig. 18). There is a reduction of the biases in the location of the mid-latitude jets when going from IPSLCM4 to CM5A-LR and CM5A-MR. The mid-latitude moist bias is also reduced when refining the horizontal grid.
For the temperature, the reduction of the global cold bias when going from CM5A-LR to -MR comes mainly from the modified tuning. However, increasing the horizontal resolution also contributes to the reduction of the mid-latitude cold bias. The stratospheric biases are also significantly reduced when going from IPSL-CM4 to -CM5, as was the case in the imposed-SST simulations.

The spatial structures of the mean SST biases are shown in Fig. 20. The global mean, which reflects the different tuning of the three model versions, is subtracted. The mean bias is of $-1.6 \mathrm{~K}$ for IPSL-CM4, $-0.95 \mathrm{~K}$ for IPSLCM5A-LR and $-0.49 \mathrm{~K}$ for IPSL-CM5A-MR. The specific cold bias in the mid-latitudes reduces when refining the grid. The three configurations show however similar regional patterns: a cold bias in the mid-latitudes, a warm bias on the eastern side of the tropical oceans, and a particularly strong cold bias in the North Atlantic.

The bias in the North Atlantic SST is associated with a strong underestimation of the Atlantic meridional overturning circulation. This underestimation is visible both in the Atlantic and global ocean meridional energy transport (Fig. 21). The transport is only slightly increased when refining the grid for IPSL-CM5A but the improvement is not systematic when comparing IPSL-CM4 and IPSLCM5A. This underestimated circulation is a robust bias of our model for which no satisfactory solution has been found so far.

For the rainfall (Fig. 22), an important deficiency of the IPSL-CM4 version was the presence of a second zone of convergence south of the equator, both in the Pacific and Atlantic ocean. This double ITCZ is a classical bias of coupled models (see e.g. Dai 2006). It is still present in the new version, both in the LR and MR configurations. The monsoon rainfall over West Africa and the Indian subcontinent did not extend sufficiently to the north in IPSLCM4. This point is slightly improved in the new version. This could be due in part to the reduction of the latitudinal biases in SST and in part to the modifications in the surface scheme. Since this question of the latitudinal extension of monsoons is rather important, this could deserve further investigations. Apart from this point, the rainfall is similar in the various configurations.

\subsection{Climate sensitivity}

We present in Fig. 23 the evolution of the mean 2-meter air temperature $\left(T_{2 \mathrm{~m}}\right)$ in a series of control (constant forcing) and $1 \% \mathrm{CO}_{2}$ experiments. In the latter, everything is kept constant in the model except the atmospheric concentration of carbon dioxide, which increases by $1 \%$ each year. The $1 \% \mathrm{CO}_{2}$ simulations start from a 1 st of January on one particular year of the control experiment taken as year 0 for the graphics. Two IPSL-CM4 simulations, using the 

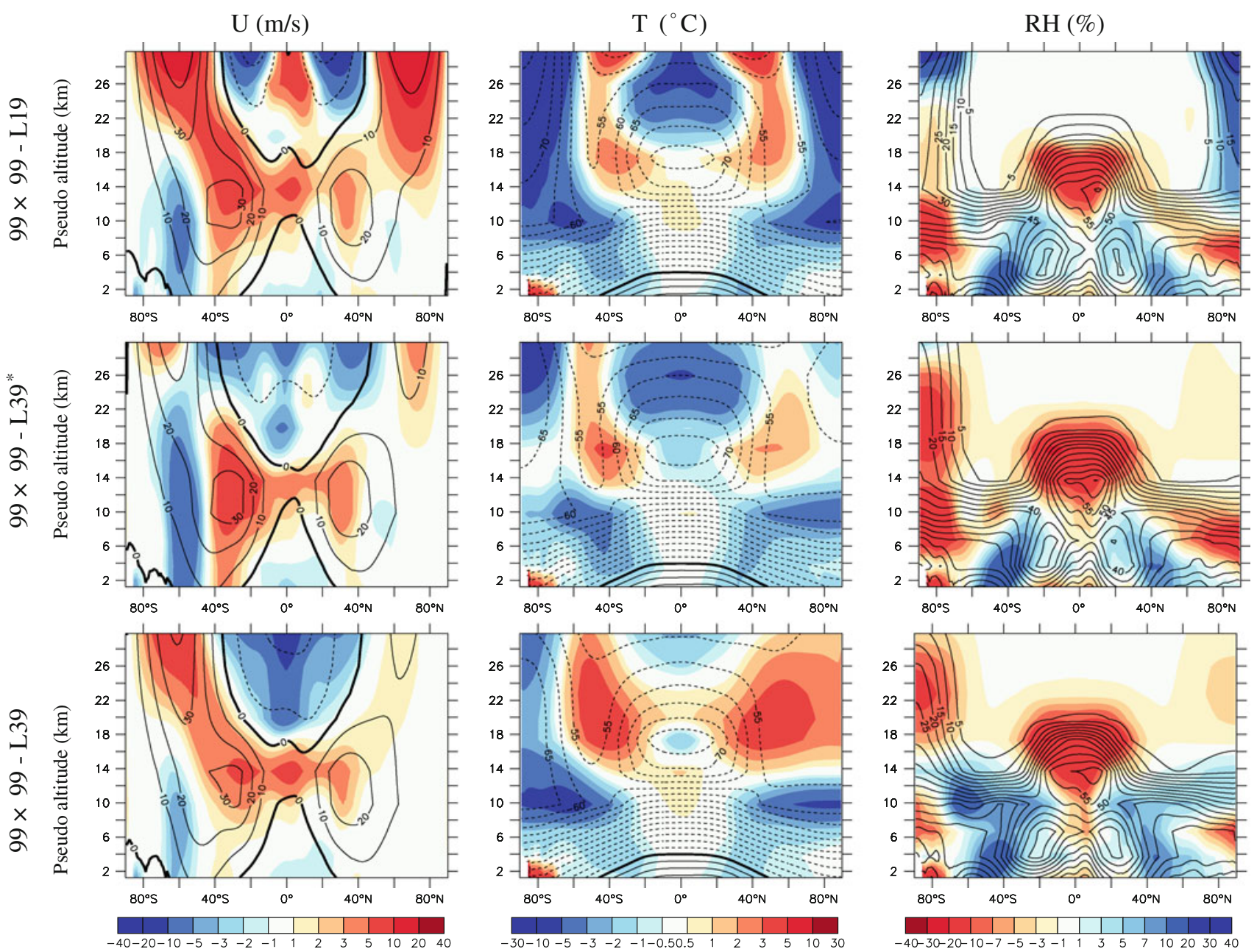

Fig. 18 Mean meridional structure of the Zonal wind (m/s, left), temperature $\left({ }^{\circ} \mathrm{C}\right.$, middle) and relative humidity for the L19, L39* and L39 forced simulations with the LR horizontal grid. The vertical axis is a pseudo-altitude $\left(-H \ln \left(p / p_{s}\right)\right.$, with $\left.H=7 \mathrm{~km}\right)$

coarsest horizontal grids $(96 \times 71$ and $96 \times 95)$, were tuned by lowering the surface albedo (by subtracting 1 and $0.9 \%$ respectively), so as to obtain an averaged global mean temperature close to that of the $144 \times 142$ configuration for the control simulations run with present-day greenhouse gases concentrations. Those simulations are called $96 \times 71 \mathrm{~B}$ and $96 \times 95 \mathrm{~B}$. The $96 \times 71 \mathrm{~B}$ simulation is still $0.5 \mathrm{~K}$ colder than $96 \times 95 \mathrm{~B}$ and $144 \times 142$ (Fig. 23a). Control simulations and climate sensitivity experiments were done with both $96 \times 71$ and $96 \times 71 \mathrm{~B}$ to check whether the tuning affects the climate sensitivity. For the IPSL-CM5A model, the tuned (lowered) ocean albedo also explains a large part of the difference between the LR and MR configurations (Fig. 23b).

Despite those changes in grid configuration, in tuning or in the mean temperature biases, the transient climate response (defined as the difference between the $1 \% \mathrm{CO}_{2}$ and control experiments at time of $\mathrm{CO}_{2}$ doubling, i.e. around year 70) is almost the same for all the model configurations as illustrated in the lower panel of Fig. 23. The ocean uptake is also comparable for all the simulations (not shown). A rigorous climate sensitivity analysis based on both $1 \% \mathrm{CO}_{2}$ and abrupt $4 \times \mathrm{CO}_{2}$ experiments shows that the climate sensitivity and feedback parameters differ by less than $10 \%$ between the IPSL-CM4, IPSL-CM5A-LR and -MR models (see Dufresne et al., this issue).

The regional distribution of global warming (left part of Fig. 24 for IPSL-CM4 and of Fig. 25 for-CM5A) also shows quite consistent results between the different versions, and reflects the usual robust aspects of climate change simulations: a stronger warming over the continents (where evaporative cooling is limited) than over oceans, a stronger warming in the (more continental) northern hemisphere than in the southern one, and in high than in low latitudes in the northern hemisphere. The simulations also show, in a rather consistent way, a weak warming in the Southern Ocean and in the North Atlantic.

The situation is a bit different for changes in the mean rainfall. Some aspects appear to be quite robust, such as the global increase of rainfall in the ITCZ/SPCZ region, and a 

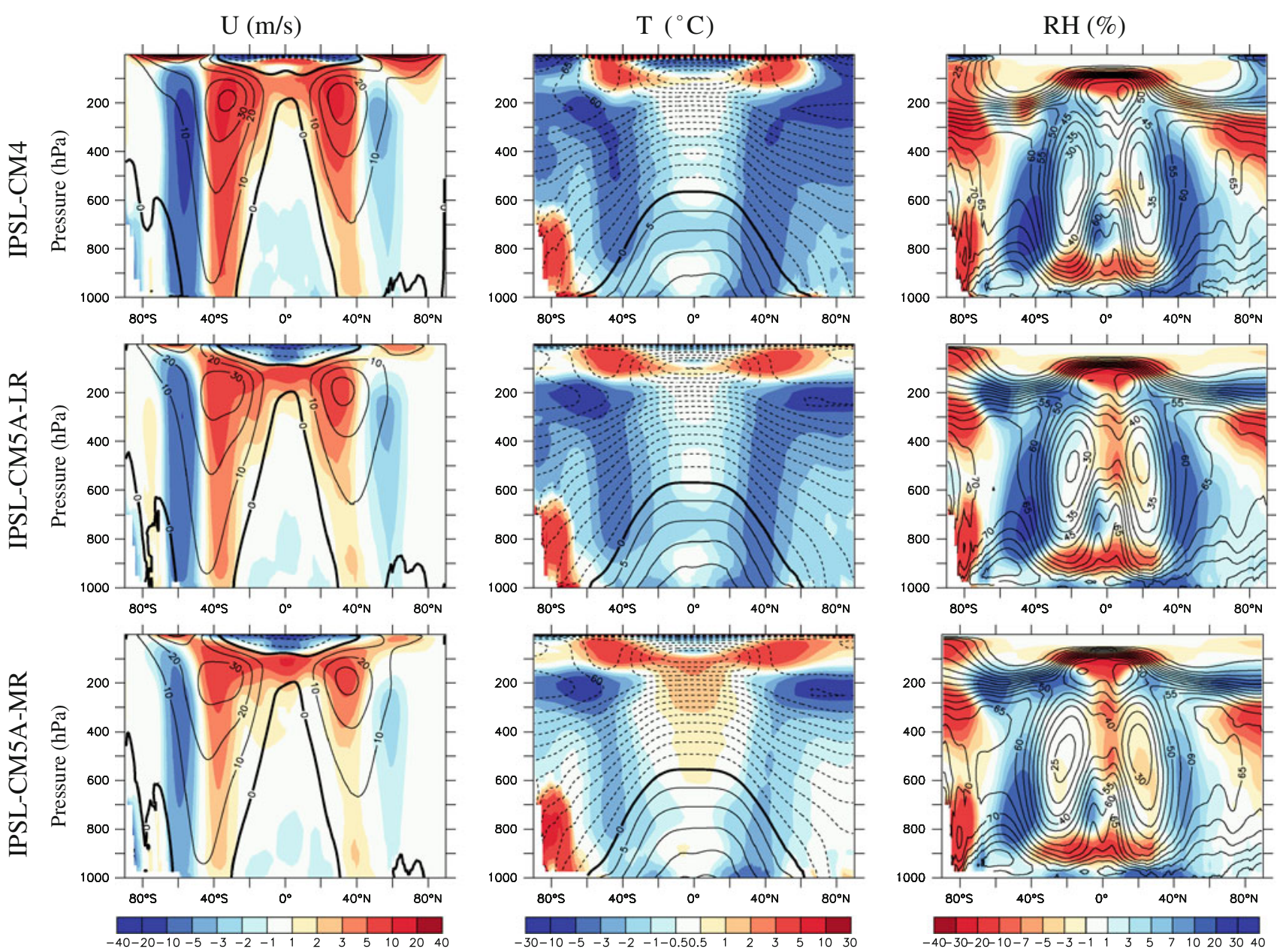

Fig. 19 Mean meridional structure of the Zonal wind $(\mathrm{m} / \mathrm{s}$, left $)$, temperature $\left({ }^{\circ} \mathrm{C}\right.$, middle $)$ and relative humidity (\%) for the IPSLCM4, IPSL-CM5A-LR and -MR simulations. The IPSL-CM4

relative drying at around 30-40 degrees latitude in both hemispheres, also a rather robust feature of CMIP3 projections (Held and Soden 2006). However, when looking at regional changes over the continents, results differ quite significantly between IPSL-CM4 and CM5A. Generally speaking, the CM4 configuration tends to predict a stronger drying (in particular over Amazonia, central Africa, India) than CM5A does, while, for each model, the results are much more consistent when varying the horizontal resolution. The differences between the CM4 and CM5A results are probably due to some significant changes in the Orchidee land-surface model between the two versions: a bug fix which had a particularly strong impact in semi-arid regions, a soil reservoir twice as deep in CM5A and the activation of the $\mathrm{CO}_{2}$ cycle which influences the Leaf Area Index (Dufresne et al., this issue). Whether one of those changes is responsible for the changes observed is a question which could deserve further investigations. simulation corresponds to a control run in present conditions while we show for IPSL-CM5A-LR and -MR the 1990-1999 decade of historical runs

\section{Conclusions}

We have explored the impact of the horizontal and vertical grid configuration of an atmospheric general circulation model on the results of both imposed-SST and coupled simulations, focusing on the representation of the mean climate and on the climate sensitivity to greenhouse gases concentration.

The refinement of the horizontal grid has a significant and systematic impact on the model biases, in particular on the latitude of the jets and on the humidity and temperature in the mid latitudes. Refining the grid in latitude rather than in longitude has a stronger impact on the latitude of the mid-latitude jets in the dynamical core experiments (Guemas and Codron 2011) and in the imposed-SST climate simulations, and a stronger impact on the reduction of the cold mid-latitude SST bias with respect to Equator in the coupled experiments. 
Fig. 20 Mean SST bias in the IPSL-CM4 and CM5A-L/MR model. The CM4 simulation is a control run while we consider the historical simulations for CM5. The mean bias is subtracted to concentrate on the structures. This mean bias is of $-1.6 \mathrm{~K}$ for IPSL-CM4, $-0.95 \mathrm{~K}$ for IPSL-CM5A-LR and -0.49 $\mathrm{K}$ for IPSL-CM5A-MR
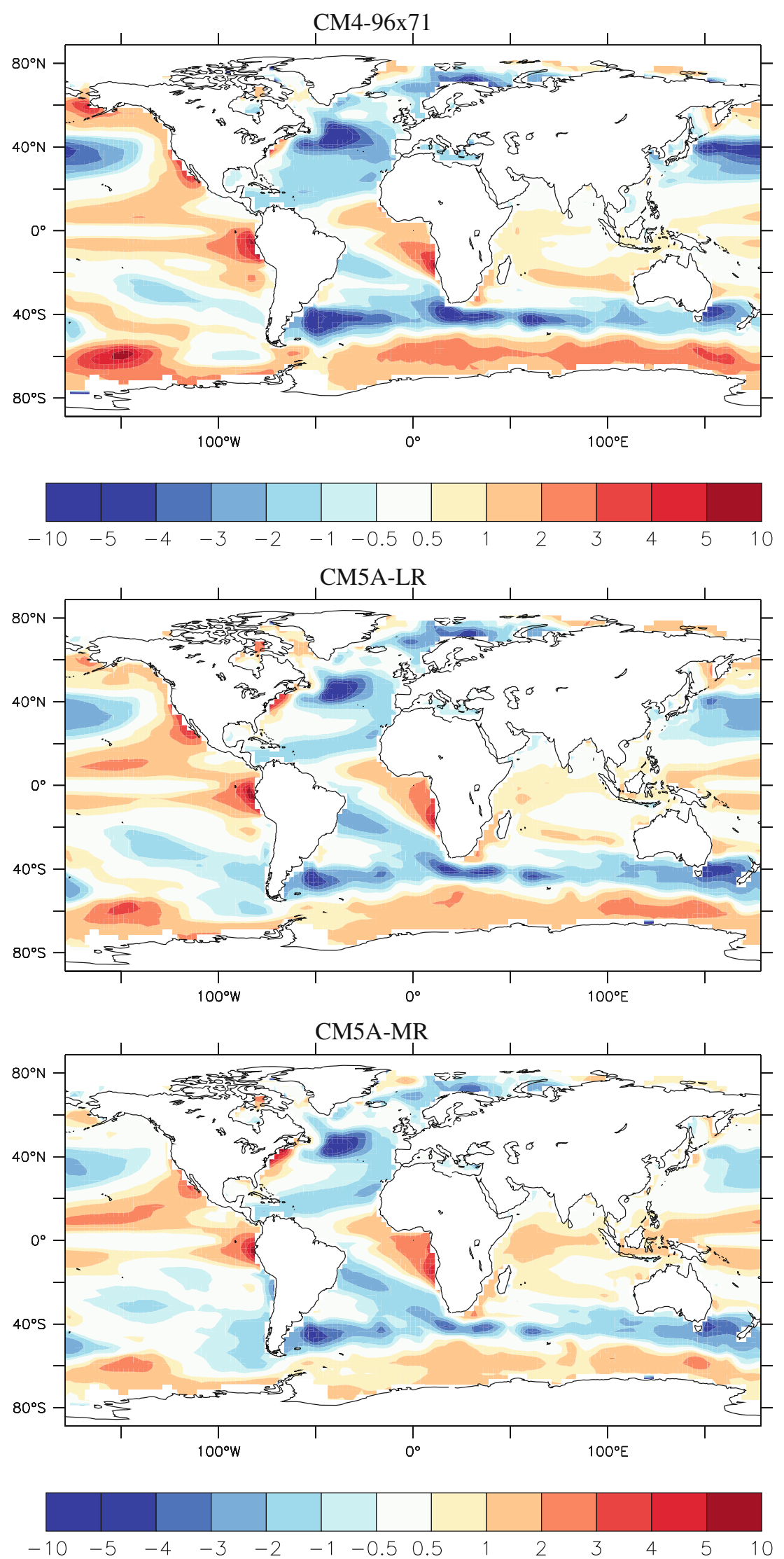
Fig. 21 Global (top) and Atlantic (bottom) total meridional energy transport by the ocean (in PW) in the IPSLCM4, IPSL-CM5A-LR and IPSL-CM5A-MR simulations. Heat transport estimates (Ganachaud and Wunsch 2003) are obtained by inversion or hydrographic data from the World ocean circulation experiment
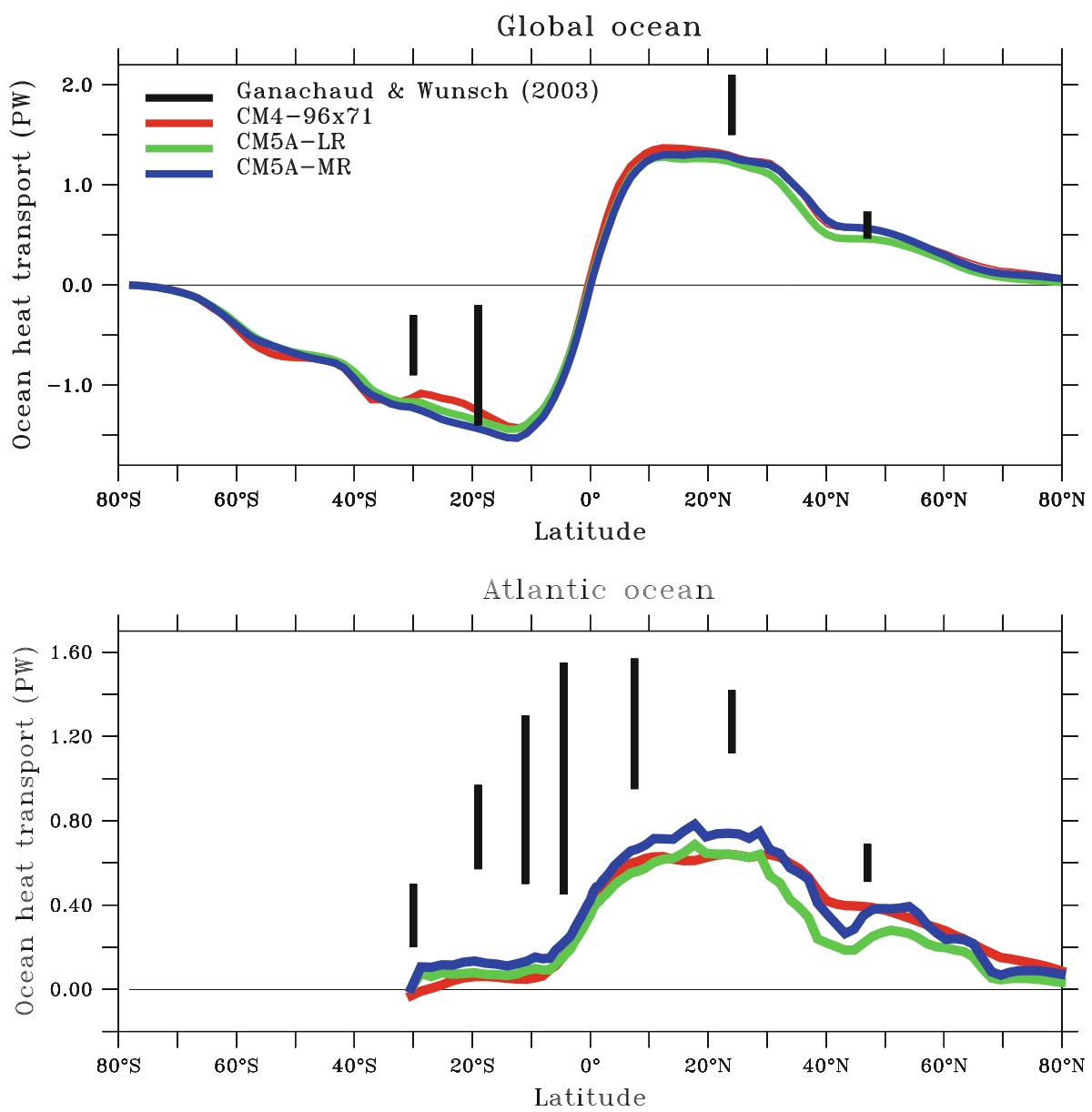

The changes of atmospheric dynamics when refining the grid are associated with significant changes in the meridional transport of heat and moisture. In mid-latitudes, grid refinement (in particular in latitude) reduces systematically a strong moist bias of the coarsest configurations, which results in less low-level clouds. In imposed-SST simulations, this decrease in cloudiness weakens the vertically integrated tropospheric radiative cooling and thus reduces the cold atmospheric bias in mid-latitudes. In coupled atmosphere-ocean simulations, the reduced cloud cover enhances the short-wave radiation at the surface in mid latitudes, and thus contributes to reduce the cold SST bias in that region. Changes in the tropical circulation are also observed when increasing the resolution in longitude that also contribute to reduce the low-level cloud cover.

Starting the explanation from the change of dynamics is somewhat arbitrary since it is possible that the changes of atmospheric transport at work in the full climate model are influenced by other processes, coming for instance from a direct sensitivity of the physical parameterizations to the grid size. However, the fact that the jet displacement mimics that observed in the idealized simulations with newtonian cooling, and that we are able to derive a complete and consistent explanation of the changes observed apart from this initial change, suggests that it could explain at least a large part of the modifications observed. This point would deserve however additional investigations, including for instance the use of idealized water-like tracers in idealized simulations with newtonian cooling.

It is shown also that the extension of the vertical grid to higher levels improves the representation of the stratospheric mean flow and of stratospheric sudden warmings.

Changing the grid configuration also has an impact on the global energy balance. Refinement of the horizontal grid results in a warmer climate in the IPSL-CM model as a consequence of the above mentioned decrease in low-level cloud cover which induces weaker (less negative) SW-CRF. The impact of refining the vertical grid is even stronger and is mainly related to changes in high-level cloudiness. The modifications are as large as $3 \mathrm{~W} / \mathrm{m}^{2}$ when refining the horizontal grid from $96 \times 71$ to $280 \times 192$, or about -6 $\mathrm{W} / \mathrm{m}^{2}$ when changing the vertical discretization from L19 to L39. In the coupled model, the model global radiation balance is restored through an increase of the global-mean nearsurface temperature, by about $1.2 \mathrm{~K}$ per $\mathrm{W} / \mathrm{m}^{2}$. 
CM4 - $96 \times 71$

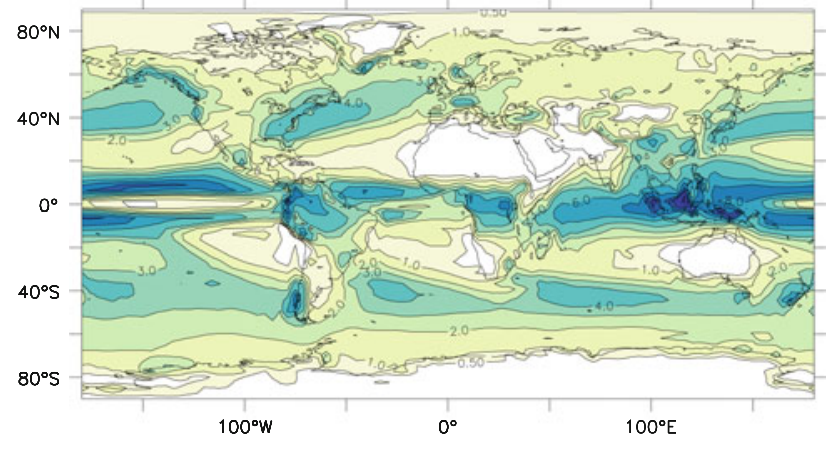

CM5A-LR
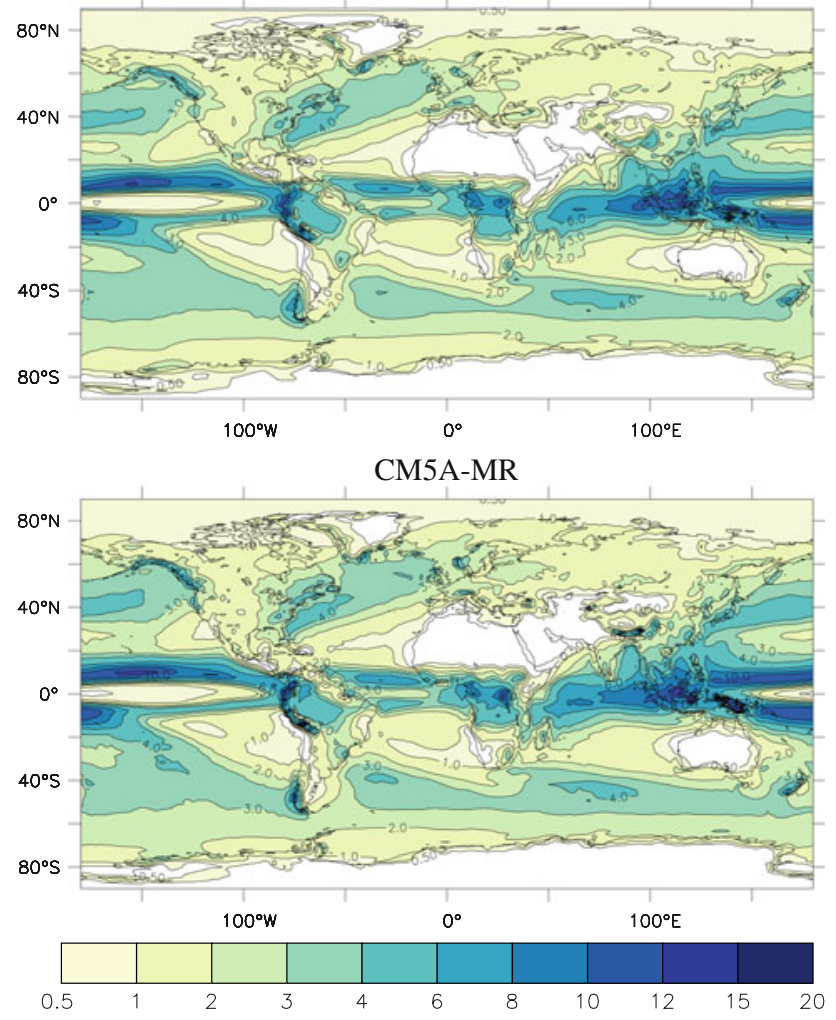

Fig. 22 Annual mean rainfall ( $\mathrm{mm} /$ day) in the control simulation with IPSL-CM4 and in the "historical simulation" for the end of the 20th century with the CM5A-LR and CM5A-MR configurations

After such configuration changes, the model must thus be tuned to compensate for the changes in energy balance. Tuning of free parameters, often unmentioned in publications, is central to climate modeling. Tuning was used here to restore the energy balance in the imposed-SST simulations and so to reduce the biases in the simulated mean surface temperature compared to present-day observations in the coupled model (the IPSL-CM5A-MR tuning is more satisfactory for that point). The tuning of the IPSL-CM5A-LR also helped to partially compensate the bias in the location of the mid-latitude jets that results from the rather coarse
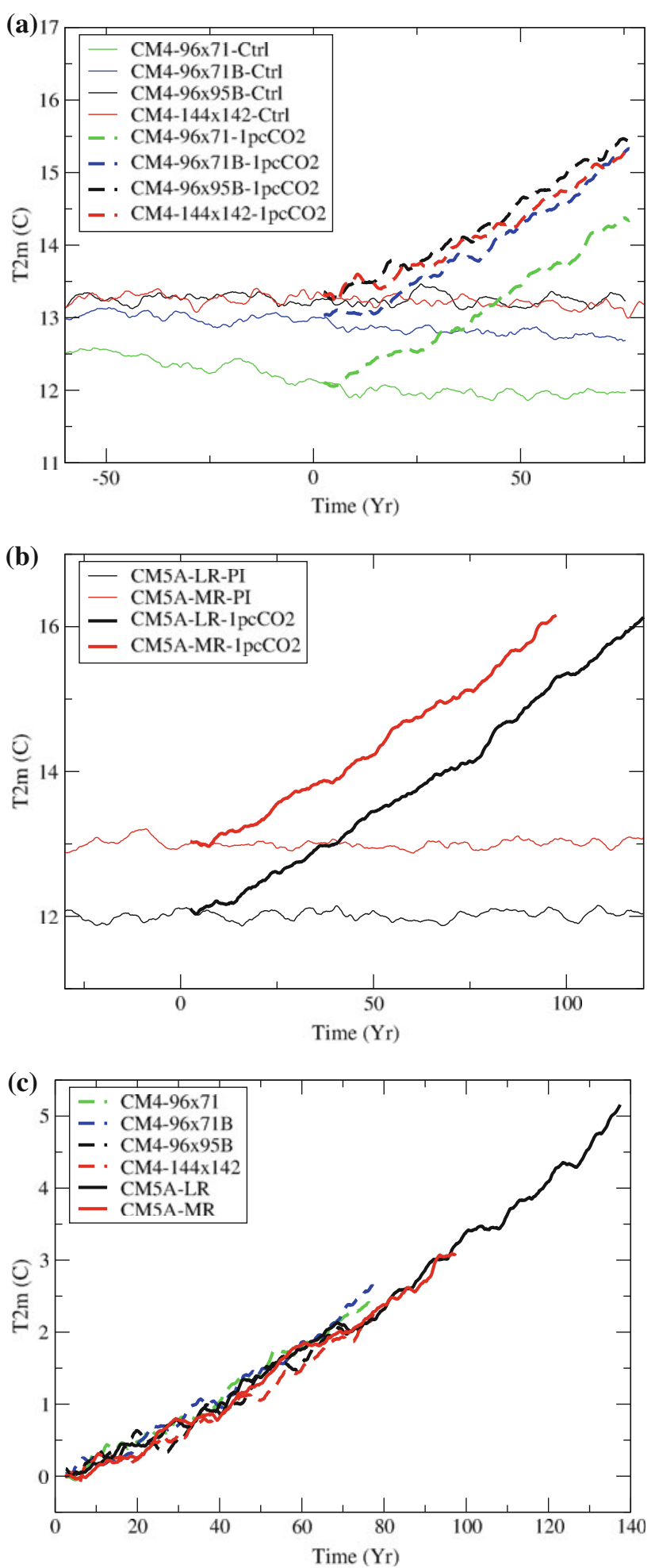

Fig. 23 Evolution of the global mean of the $2 \mathrm{~m}$ air temperature in control and $1 \% \mathrm{CO}_{2}$ experiments with the IPSL-CM4 and IPSLCM5A model with various grid configurations. a, b show the temperature itself (in ${ }^{\circ} \mathrm{C}$ ) while $\mathbf{c}$ shows the difference between the $1 \% \mathrm{CO}_{2}$ experiment and the corresponding control simulation 
Temperature change $(\mathrm{K})$

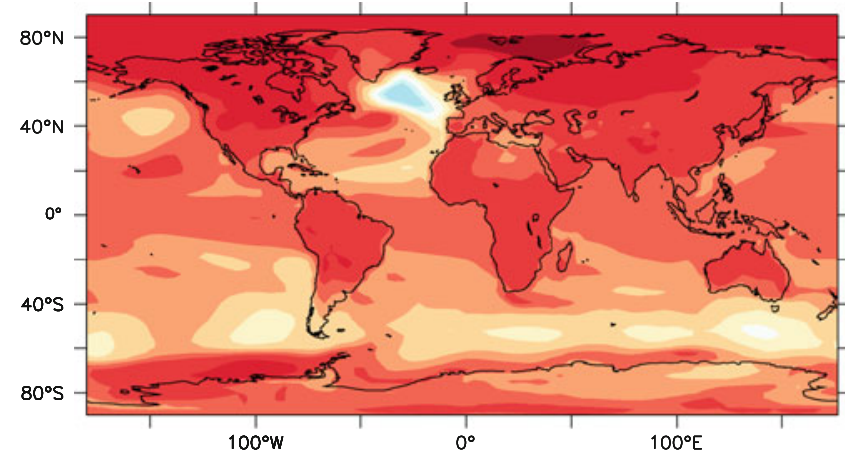

CM4-96x71

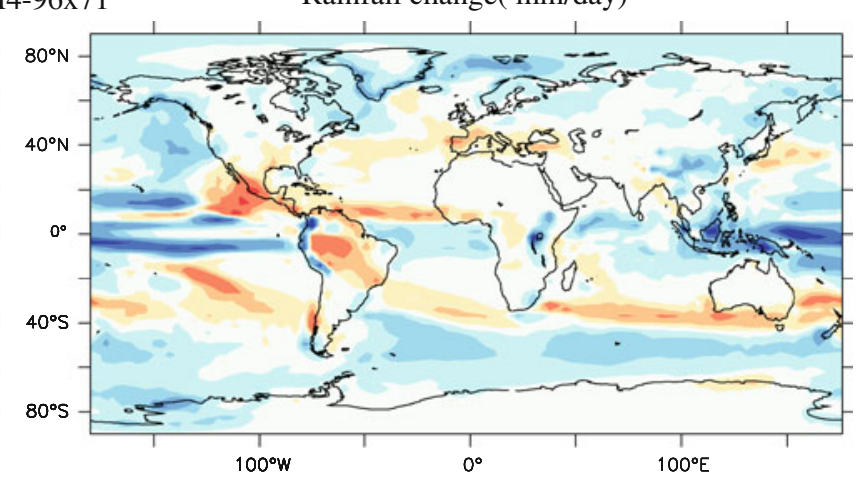

CM4-96x71B
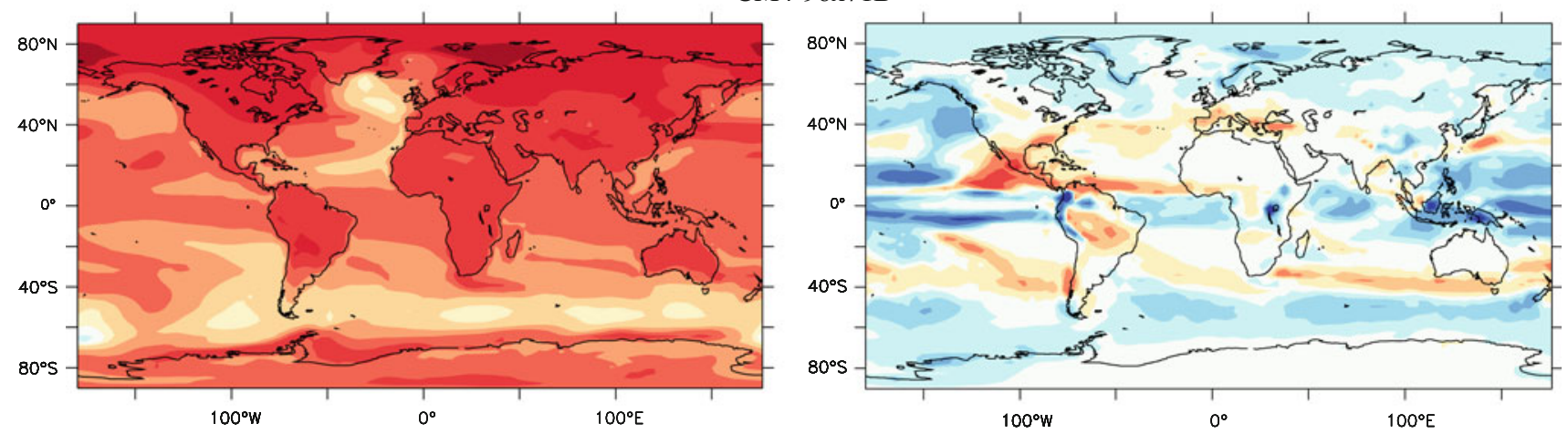

CM4-96x95B
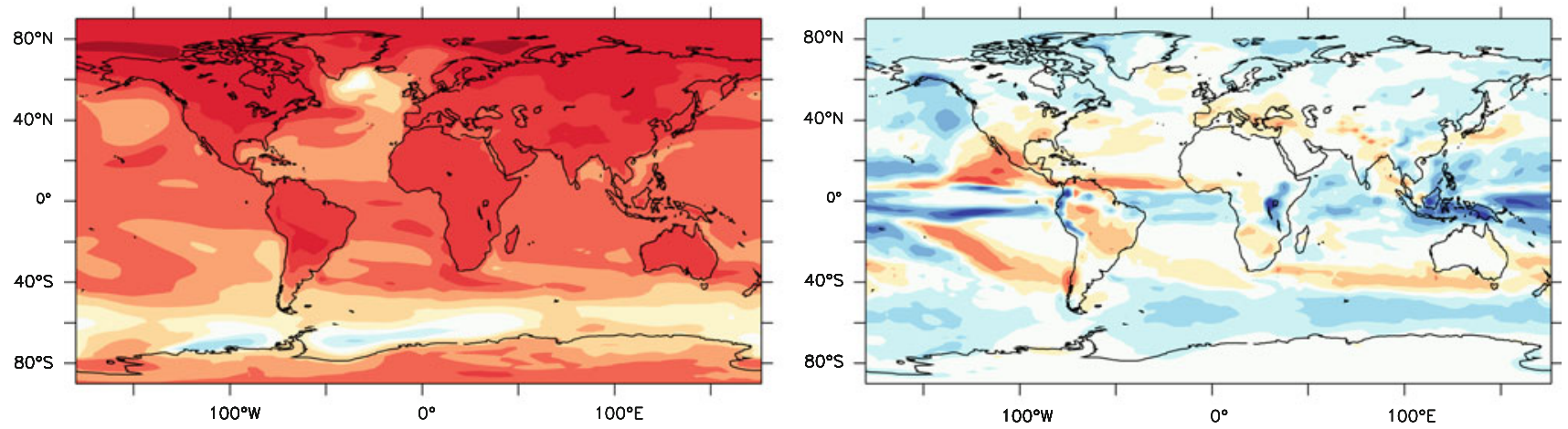
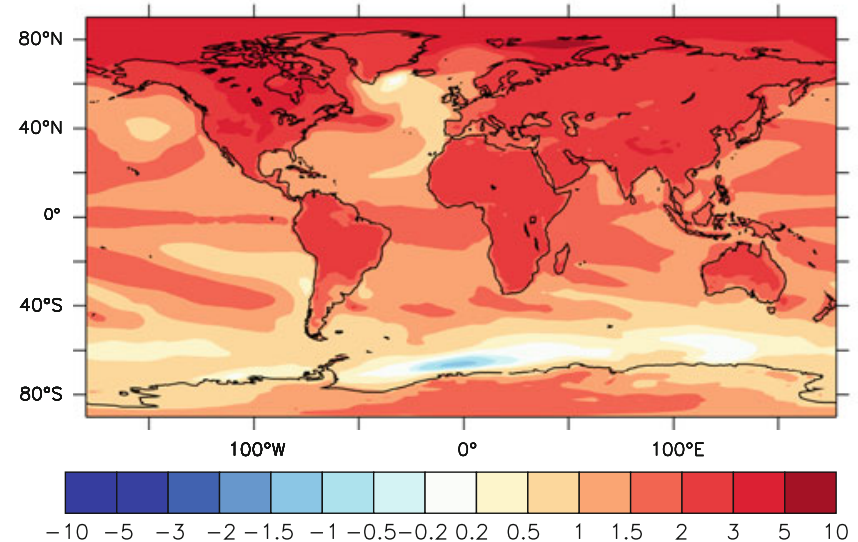

Fig. $242 \mathrm{~m}$ air temperature (K, left column) and precipitation $(\mathrm{mm} /$ day, right column) changes in $1 \% \mathrm{CO}_{2}$ experiments with various configurations of IPSL-CM4, showing the difference between the

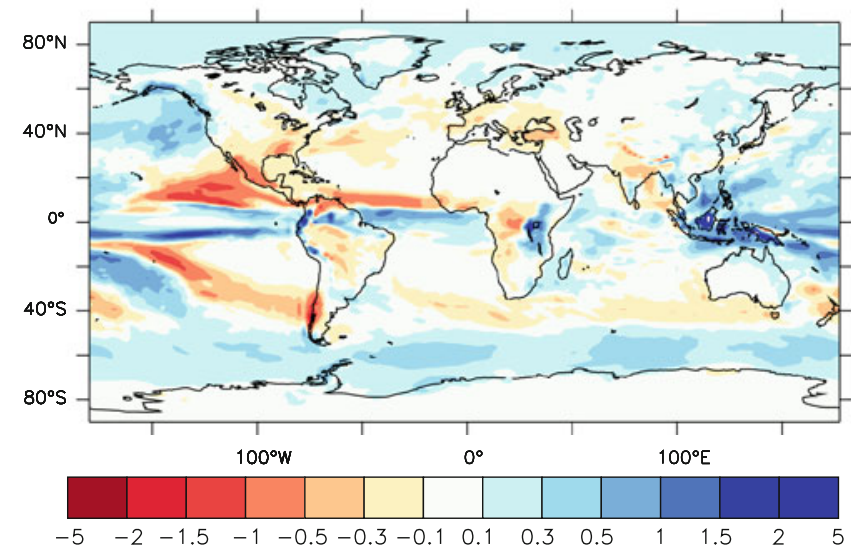

average variable for the last 30 years (year 51-80) of the $1 \% \mathrm{CO}_{2}$ simulation minus the value of the control simulations for the same period 

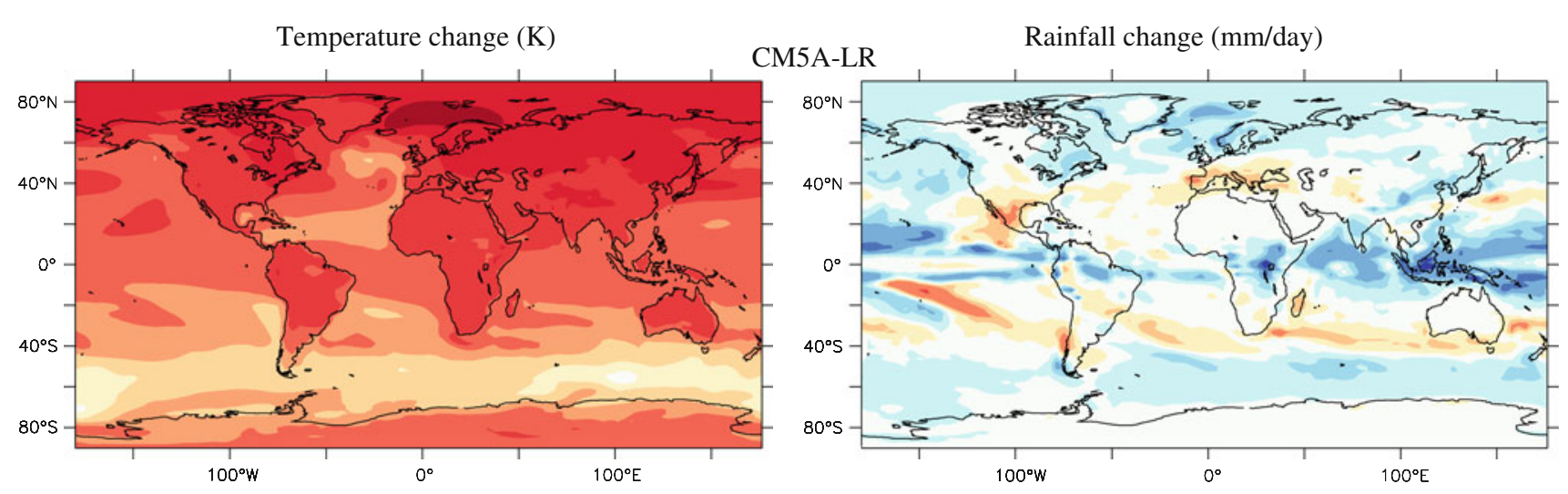

CM5A-MR
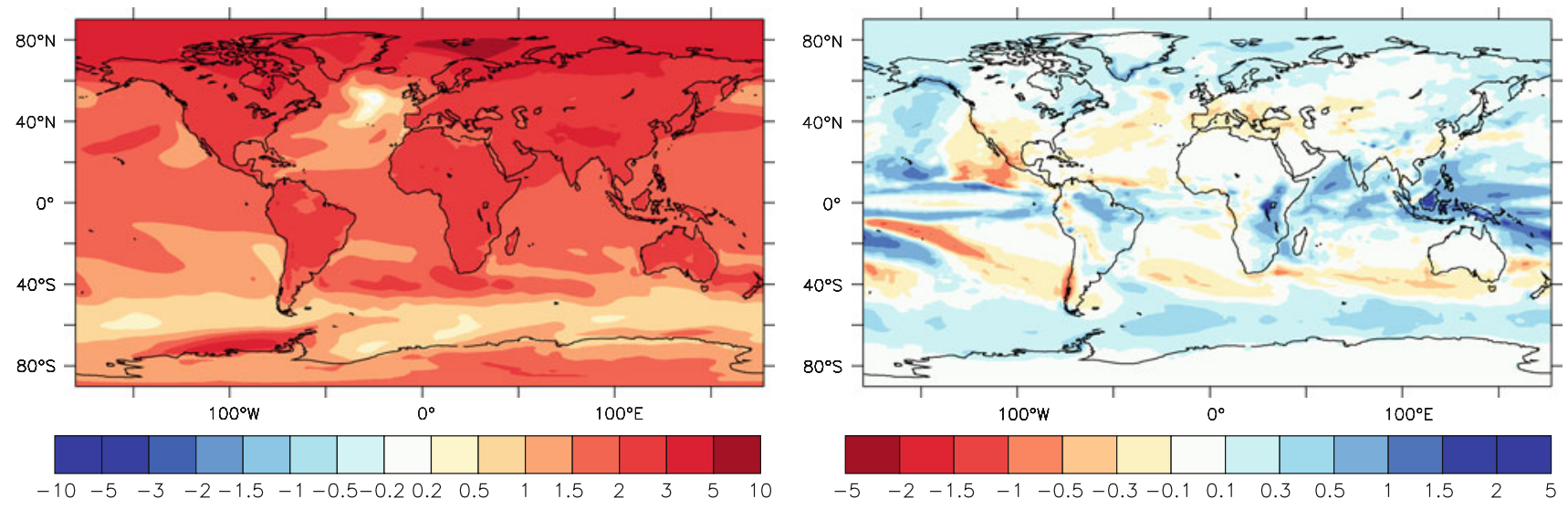

Fig. $252 \mathrm{~m}$ air temperature (K, left column) and precipitation $(\mathrm{mm} /$ day, right column) changes in $1 \% \mathrm{CO}_{2}$ experiments with IPSLCM5A-LR and -MR, showing the difference between the average

horizontal resolution. Generally however, tuning did not help reduce several major robust biases of the model such as the warm SST biases at the eastern side of tropical oceans, the double ITCZ structure or the underestimated meridional ocean heat transport. Tuning with respect to present-day observations does not guarantee either a better representation of the climate sensitivity to greenhouse gases.

Despite significant changes in configuration which result in a significantly modified representation of the present-day climate, the various IPSL-CM configurations presented here show a very similar sensitivity to the concentration of greenhouse gases. This is an important result for climate change studies: if grid refinement allows to improve the realism of climate models in their representation of the present-day climate, it seems of secondary importance for many aspects of climate projections.

The above result must be contrasted with the strong reduction of climate sensitivity obtained in the CM5B version of the IPSL coupled model, when changing the parameterizations of clouds and convection (Hourdin et al. 2012). The simple conclusion could thus be that grid refinement, both in the vertical and horizontal affects the climate sensitivity to a lesser extent than changes in clouds

variable for the last 30 years (year 51-80) of the $1 \% \mathrm{CO}_{2}$ simulation minus the value of the control simulations for the same period

parameterizations. It must be kept in mind, however, that a careful retuning of the model was done when changing the vertical grid, in such a way that the final net CRF in LMDZ5A is very close to that of the previous LMDZ4 L19 version (lower mid panel in Fig. 16). Some recent results suggest that a different tuning of the same set of cloud parameterizations could modify as well the sensitivity to greenhouse gases (Brient and Bony 2012).

Acknowledgments The work presented in this paper has largely benefited from the work of our colleagues of the IPSL Climate Modelling Centre. The research leading to these results was supported by CNRS, the INSU-LEFE French Program under the MissTerre. It also received support from the COMBINE EU project (EC IP, Grant Agreement number 226520). This work also benefited of the HPC resources of CCRT and IDRIS made available by GENCI (Grand Equipement National de Calcul Intensif). The discussion on tuning and confidence in climate change projections benefited from meetings with epistemologists from Centre Koyré as part of the french ANR project "ClimaConf". We also would like to thank the anonymous referees for their constructive and helpful remarks on this long manuscript.

Open Access This article is distributed under the terms of the Creative Commons Attribution License which permits any use, distribution, and reproduction in any medium, provided the original author(s) and the source are credited. 


\section{References}

Aumont O, Bopp L (2006) Globalizing results from ocean in situ iron fertilization studies. Global Biogeochem Cycles 20:GB2017. doi: $10.1029 / 2005 \mathrm{~GB} 002591$

Baldwin MP, Dunkerton TJ (1999) Propagation of the Arctic oscillation from the stratosphere to the troposphere. J Geophys Res 1043:30,937-30,946. doi:10.1029/1999JD900445

Baldwin MP, Gray LJ, Dunkerton TJ, Hamilton K, Haynes PH, Randel WJ, Holton JR, Alexander MJ, Hirota I, Horinouchi T, Jones DBA, Kinnersley JS, Marquardt C, Sato K, Takahashi M (2001) The quasi-biennial oscillation. Rev Geophys 39:179-230. doi:10.1029/1999RG000073

Bony S, Emanuel KA (2001) A parameterization of the cloudiness associated with cumulus convection; evaluation using TOGA COARE data. J Atmos Sci 58:3158-3183

Bony S, Dufresne JL, Le Treut H, Morcrette JJ, Senior C (2004) On dynamic and thermodynamic components of cloud changes. Clim Dyn 22:71-86. doi:10.1007/s00382-003-0369-6

Braconnot P, Hourdin F, Bony S, Dufresne JL, Grandpeix JY, Marti O (2007) Impact of different convective cloud schemes on the simulation of the tropical seasonal cycle in a coupled oceanatmosphere model. Clim Dyn 29:501-520

Brient F, Bony S (2012) Interpretation of the positive low-cloud feedback predicted by a climate model under global warming. Clim Dyn 6. doi:10.1007/s00382-011-1279-7

Byrkjedal O, Esau I, Kvamsto NG (2008) Sensitivity of simulated wintertime Arctic atmosphere to vertical resolution in the arpege/ifs model. Clim Dyn 30(7-8):687

Charney JG, Drazin PG (1961) Propagation of planetary-scale disturbances from the lower into the upper atmosphere. J Geophys Res 66:83-109. doi:10.1029/JZ066i001p00083

Conkright ME, Locarnini R, Garcia H, O'Brien T, Boyer T, Stephens C, Antonov J (2002) World ocean atlas 2001: objective analyses, data statistics and figures cd-rom documentation. National Oceanographic Data Center Internal Report 17, US Department of Commerce

Cunningham SA, Alderson SG, King BA, Brandon MA (2003) Transport and variability of the Antarctic circumpolar current in drake passage. J Geophys Res (Oceans) 108:8084. doi:10.1029/ 2001JC001147

Dai A (2006) Precipitation characteristics in eighteen coupled climate models. J Clim 19:4605-4630B. doi:10.1175/JCLI3884.1

de Rosnay P, Polcher J, Bruen M, Laval K (2002) Impact of a physically based soil water flow and soil-plant interaction representation for modeling large scale land surface processes. J Geophys Res 107. doi:10.1029/2001JD000634

Deardorff JW (1966) The counter-gradient heat-flux in the lower atmosphere and in the laboratory. J Atmos Sci 23:503-506

Déqué M, Dreverton C, Braun A, Cariolle D (1994) The Arpege/IFS atmosphere model: a contribution to the french community climate modelling. Clim Dyn 10:249-266

Emanuel KA (1991) A scheme for representing cumulus convection in large-scale models. J Atmos Sci 48:2313-2335

Ganachaud A, Wunsch C (2003) Large-scale ocean heat and freshwater transports during the world ocean circulation experiment. J Clim 16:696-705. doi:10.1175/1520-0442(2003)016 $<0696$ :LSOHAF>2.0.CO;2

Guemas V, Codron F (2011) Differing impacts of resolution changes in latitude and longitude on the mid-latitudes in the LMDZ GCM. J Clim. doi:10.1175/2011JCLI4093.1

Hack JJ, Caron JM, Danabasoglu G, Oleson KW, Bitz C, Truesdale JE (2006) Ccsm-cam3 climate simulation sensitivity to changes in horizontal resolution. J Clim 19:2267-2289

Held IM, Soden BJ (2006) Robust responses of the hydrological cycle to global warming. J Clim 19:5686-5699. doi:10.1175/JCLI3990.1
Held IM, Suarez MJ (1994) A proposal of the dynamical cores of atmospehric general circulation models. Bull Am Meteor Soc 75(10):1825-1830

Heymsfield AJ, Donner LJ (1990) A scheme for parameterizing icecloud water content in general circulation models. J Atmos Sci 47:1865-1877. doi:10.1175/1520-0469(1990)047<1865:ASFPIC> 2.0.CO; 2

Hourdin F, Musat I, Bony S, Braconnot P, Codron F, Dufresne JL, Fairhead L, Filiberti MA, Friedlingstein P, Grandpeix JY, Krinner G, Levan P, Li ZX, Lott F (2006) The LMDZ4 general circulation model: climate performance and sensitivity to parametrized physics with emphasis on tropical convection. Clim Dyn 27:787-813. doi:10.1007/s00382-006-0158-0

Hourdin F, Grandpeix JY, Rio C, Bony S, Jam A, Cheruy F, Rochetin N, Fairhead L, Idelkadi A, Musat I, Dufresne JL, Lahellec A, Lefebvre MP, Roehrig R (2012) LMDZ5B: the atmospheric component of the ipsl climate model with revisited parameterizations for clouds and convection. doi:10.1007/s00382-012-1343-y

Huffman GJ, Adler RF, Morrissey MM, Bolvin DT, Curtis S, Joyce R, McGavock B, Susskind J (2001) Global precipitation at one-degree daily resolution from multisatellite observations. J Hydrometeorol 2:36-50. doi:10.1175/1525-7541(2001)002 $<0036$ :GPAODD $>2.0$.CO;2

Hurrell JW, Hack JJ, Shea D, Caron JM, Rosinski J (2008) A new sea surface temperature and sea ice boundary dataset for the community atmosphere model. J Clim 21:5145-5153. doi: 10.1175/2008JCLI2292.1

Jourdain L, Bekki S, Lott F, Lefèvre F (2008) The coupled chemistryclimate model LMDZ-REPROBUS: description and evaluation of a transient simulation of the period 1980-1999. Ann Geophys 26:1391-1413. doi:10.5194/angeo-26-1391-2008

Kiehl JT, Williamson DL (1991) Dependence of cloud amount on horizontal resolution in the National Center for Atmospheric Research Community Climate Model. J Geophys Res 96(D6): 10,955-10,980. doi:10.1029/91JD00164

Kobayashi C, Sugi M (2004) Impact of horizontal resolution on the simulation of the asian summer monsoon and tropical cyclones in the jma global model. Clim Dyn 23:165-176

Krinner G, Viovy N, de Noblet-Ducoudré N, Ogée J, Polcher J, Friedlingstein P, Ciais P, Sitch S, Prentice C (2005) A dynamic global vegetation model for studies of the coupled atmospherebiosphere system. Glob Biogeochem Cyc 19:GB1015. doi: 10.1029/2003GB002199

Laval K, Sadourny R, Serafini Y (1981) Land surface processes in a simplified general circulation model. Geophys Astrophys Fluid Dyn 17:129-150

Loeb NG, Wielicki BA, Doelling DR, Smith GL, Keyes DF, Kato S, Manalo-Smith N, Wong T (2009) Toward optimal closure of the earth's top-of-atmosphere radiation budget. J Clim 22(3):748766. doi:10.1175/2008JCLI2637.1

Lott F (1999) Alleviation of stationary biases in a GCM through a mountain drag parameterization scheme and a simple representation of mountain lift forces. Mon Weather Rev 127:788-801. doi:10.1175/1520-0493(1999)127<0788:AOSBIA > 2.0.CO;2

Lott F, Fairhead L, Hourdin F, Levan P (2005) The stratospheric version of LMDz: dynamical climatologies, arctic oscillation, and impact on the surface climate. Clim Dyn 25:851-868

Louis JF (1979) A parametric model of vertical eddy fluxes in the atmosphere. Boundary-Layer Meteorol 17:187-202

Marti O, Braconnot P, Dufresne J, Bellier J, Benshila R, Bony S, Brockmann P, Cadule P, Caubel A, Codron F, de Noblet N, Denvil S, Fairhead L, Fichefet T, Foujols M, Friedlingstein P, Goosse H, Grandpeix J, Guilyardi E, Hourdin F, Idelkadi A, Kageyama M, Krinner G, Lévy C, Madec G, Mignot J, Musat I, Swingedouw D, Talandier C (2010) Key features of the IPSL 
ocean atmosphere model and its sensitivity to atmospheric resolution. Clim Dyn 34:1-26. doi:10.1007/s00382-009-0640-6

Meehl GA, Covey C, Delworth T, Latif M, McAvaney B, Mitchell JFB, Stouffer RJ (2007) The WCRP CMIP3 multi-model dataset: a new era in climate change research. Bull Am Meteorol Soc 88(9):1383-1394. doi:10.1175/BAMS-88-9-1383

Morcrette J (1991) Radiation and cloud radiative properties in the European centre for medium range weather forecasts forecasting system. J Geophys Res 96:9121-9132

Navarra A, Gualdi S, Masina S, Behera S, Luo J-J, Masson S, Guilyardi E, Delecluse P, Yamagata T (2008) Atmospheric horizontal resolution affects tropical climate variability in coupled models. J Clim 21(4):730-750. doi:10.1175/2007JCLI1406.1

Nikulin G, Lott F (2010) On the time-scales of the downward propagation and of the tropospheric planetary wave response to the stratospheric circulation. Ann Geophys 28:339-351. doi: 10.5194/angeo-28-339-2010

Pope VD, Stratton RA (2002) The processes governing horizontal resolution sensitivity in a climate model. J Clim 14:3065-3085

Raval A, Ramanathan V (1989) Observational determination of the greenhouse effect. Nature 342:758-761. doi:10.1038/342758a0

Roeckner E, Brokopf R, Esch M, Giorgetta M, Hagemann S, Kornblueth L, Manzini E, Schlese U, Schulzweida U (2006) Sensitivity of simulated climate to horizontal and vertical resolution in the echam5 atmosphere model. J Clim 19:3771-3791
Sadourny R (1975) The dynamics of finite-difference models of the shallow-water equations. J Atmos Sci 32:680-689

Sadourny R, Laval K (1984) January and July performance of the LMD general circulation model. In: Berger A, Nicolis C (eds) New perspectives in climate modeling, Elsevier, Amsterdam, pp 173-197

Sarmiento JL, Gruber N, Brzezinski MA, Dunne JP (2004) Highlatitude controls of thermocline nutrients and low latitude biological productivity. Nature 427:56-60

Sundqvist H (1978) A parameterization scheme for non-convective condensation including prediction of cloud water content. Q J R Meteorol Soc 104:677-690. doi:10.1002/qj.49710444110

Swingedouw D, Braconnot P, Delecluse P, Guilyardi E, Marti O (2007) The impact of global freshwater forcing on the thermohaline circulation: adjustment of North Atlantic convection sites in a CGCM. Clim Dyn 28:291-305. doi:10.1007/s00382-0060171-3

Taylor KE, Stouffer RJ, Meehl GA (2012) An overview of CMIP5 and the experiment design. Bull Am Meteorol Soc. doi: 10.1175/BAMS-D-11-00094.1

Williamson DL, Kiehl JT, Hack JJ (1995) Climate sensitivity of the NCAR Community Climate Model (CCM2) to horizontal resolution. Clim Dyn 11:377-397 